\title{
SPLITTING FIELDS OF CHARACTERISTIC POLYNOMIALS OF RANDOM ELEMENTS IN ARITHMETIC GROUPS
}

\author{
F. JOUVE, E. KOWALSKI, AND DAVID ZYWINA
}

\begin{abstract}
We discuss rather systematically the principle, implicit in earlier works, that for a "random" element in an arithmetic subgroup of a (split, say) reductive algebraic group over a number field, the splitting field of the characteristic polynomial, computed using any faitfhful representation, has Galois group isomorphic to the Weyl group of the underlying algebraic group. Besides tools such as the large sieve, which we had already used, we introduce some probabilistic ideas (large deviation estimates for finite Markov chains) and the general case involves a more precise understanding of the way Frobenius conjugacy classes are computed for such splitting fields (which is related to a map between regular elements of a finite group of Lie type and conjugacy classes in the Weyl group which had been considered earlier by Carter and Fulman for other purposes; we show in particular that the values of this map are equidistributed).
\end{abstract}

\section{INTRODUCTION}

In earlier works, in particular [JKZ], we have considered particular cases of the following "principle": if $g$ is a "random" rational element in a connected split reductive group G over Q, embedded in some $\mathrm{GL}(m)$, then the splitting field of the characteristic polynomial of $g$ should have Galois group isomorphic to the Weyl group of $\mathbf{G}$.

In this paper, we consider this question in much greater generality than previously. We are thus led to replace somewhat ad hoc arguments with more intrinsic constructions, in particular in two areas: (1) in characterizing the splitting field of the polynomials we construct, which we relate to splitting fields of tori; (2) in the understanding of the situation over finite fields, which is required for the sieve argument we use to obtain strong bounds on the probability of having a Galois group smaller than expected. Moreover, to handle the reduction to simply-connected groups, we need as input some ideas from Markov chains (in particular, some large deviation estimates for finite Markov chains).

Let $k$ be a number field and denote by $\mathbf{Z}_{k}$ its ring of integers. Let $\mathbf{G}$ be a connected linear algebraic group defined over $k$. We may view it as a matrix group by fixing a faithful embedding $\rho: \mathbf{G} \hookrightarrow \mathbf{G L}(m)$ defined over $k$. For each $g \in \mathbf{G}(k)$, let $k_{g}$ be the splitting field over $k$ of the characteristic polynomial $\operatorname{det}(T-\rho(g)) \in k[T]$. The goal of this paper is to describe the Galois group $\operatorname{Gal}\left(k_{g} / k\right)$ for a "random" $g$ in terms of the geometry of $\mathbf{G}$.

We will only consider those $g$ belonging to a fixed arithmetic subgroup $\Gamma$ of $\mathbf{G}$. Recall that an arithmetic subgroup of $\mathbf{G}$ is a subgroup $\Gamma$ of $\mathbf{G}(k)$ for which $\rho(\Gamma)$ is commensurable with $\rho(\mathbf{G}(k)) \cap$ $\operatorname{GL}\left(m, \mathbf{Z}_{k}\right)$; this definition is independent of $\rho$. We shall assume that our arithmetic group $\Gamma$ is Zariski dense in $\mathbf{G}$ (otherwise the structure of the Galois groups Gal $\left(k_{g} / k\right)$ should be governed by a smaller algebraic group).

Our notion of "random" in this paper is to view $\Gamma$ as the vertices of a Cayley graph and perform a long random walk on this graph. First choose a finite set $S$ that generates the group $\Gamma$ (arithmetic groups are finitely generated, see [PR, Th. $4.17(2)]$ ), such that $S$ is symmetric, i.e., $S=S^{-1}$.

2000 Mathematics Subject Classification. 11R32, 11N35, 11N36, 20G30 (Primary); 11C08, 60J10 (Secondary).

Key words and phrases. Algebraic groups, arithmetic groups, Weyl group, characteristic polynomial, large sieve, random walk on finite groups. 
We then have a Cayley graph associated to the pair $(\Gamma, S)$ : the vertices are the elements of $\Gamma$ and there is an edge connecting the vertices $g_{1}$ and $g_{2} \in \Gamma$ if and only if $g_{1} g_{2}^{-1}$ belongs to $S$ (note we allow $1 \in S$, in which case the graph has self-loops at each vertex). This graph is regular of degree $|S|$. Starting at the vertex $1 \in \Gamma$ of our graph, we take a random walk by repeatedly following one of the $|S|$ edges emanating from the current vertex with equal probability. More precisely, for each $n \geqslant 1$, we will choose a random element $s_{n}$ of $S$ (with uniform distribution); this gives a walk $X_{0}=1, X_{1}=s_{1}, X_{2}=s_{1} s_{2}, X_{3}=s_{1} s_{2} s_{3}, X_{4}=s_{1} s_{2} s_{3} s_{4}, \ldots$

Theorem 1.1. Let $\mathbf{G}$ be a reductive group defined over a number field $k$, and fix a faithful representation $\rho: \mathbf{G} \hookrightarrow \mathrm{GL}(m)$ defined over $k$. Let $\Gamma \subseteq \mathbf{G}(k)$ be an arithmetic subgroup of $\mathbf{G}$ and assume that it is Zariski dense in $\mathbf{G}$. Let $S$ be a finite symmetric set of generators for $\Gamma$ such that $1 \in S$. For any $w=\left(s_{1}, \ldots, s_{n}\right) \in S^{n}$, let $k_{w} / k$ be the splitting field of the characteristic polynomial

$$
\operatorname{det}\left(T-\rho\left(s_{1} \cdots s_{n}\right)\right) \in k[T]
$$

over $k$. Then there is a finite group $\Pi(\mathbf{G})$ which contains the Weyl group $W(\mathbf{G})$ as a normal subgroup such that the following hold:

(i) The Galois group $\operatorname{Gal}\left(k_{w} / k\right)$ is always isomorphic to a subquotient of $\Pi(\mathbf{G})$.

(ii) We have

$$
\lim _{n \rightarrow \infty} \frac{\left|\left\{w=\left(s_{1}, \ldots, s_{n}\right) \in S^{n}: \operatorname{Gal}\left(k_{w} / k\right) \cong \Pi(\mathbf{G})\right\}\right|}{\left|S^{n}\right|}=1 .
$$

(iii) If $\mathbf{G}$ is semisimple, then there exists a constant $c>1$ such that

$$
\frac{\left|\left\{w=\left(s_{1}, \ldots, s_{n}\right) \in S^{n}: \operatorname{Gal}\left(k_{w} / k\right) \cong \Pi(\mathbf{G})\right\}\right|}{\left|S^{n}\right|}=1+O\left(c^{-n}\right)
$$

for all $n \geqslant 1$.

(iv) Let $\bar{k}$ be an algebraic closure of $k$ and let $k_{\mathbf{G}}$ be the intersection of all the extensions $K \subseteq \bar{k}$ of $k$ for which $\mathbf{G}_{K}$ is split. There exists a constant $c>1$ such that

$$
\frac{\left|\left\{w=\left(s_{1}, \ldots, s_{n}\right) \in S^{n}: \operatorname{Gal}\left(k_{\mathbf{G}} k_{w} / k_{\mathbf{G}}\right) \cong W(\mathbf{G})\right\}\right|}{\left|S^{n}\right|}=1+O\left(c^{-n}\right)
$$

for all $n \geqslant 1$.

The constants $c$ and the implied constants depend only on $\mathbf{G}$ and the set $S$.

We shall explicitly describe the group $\Pi(\mathbf{G})$ in $\S 2$. If we assume that $\mathbf{G}$ is split, then $k_{\mathbf{G}}=k$ and $\Pi(\mathbf{G})=W(\mathbf{G})$. See Theorem 6.1 for a more general version where we allow different distributions of the steps $s_{n}$ and a general connected linear algebraic group $\mathbf{G}$ over $k$.

Example 1.2. Here are some illustrations of our theorem.

(1) Let $\mathbf{G}$ be either $\operatorname{SL}(n)$ or $\operatorname{Sp}(2 g)$ where $n \geqslant 2$ and $g \geqslant 1$. We may identify $\mathbf{G}$ as a matrix group via the natural representation into $\mathrm{GL}(n)$ or $\mathrm{GL}(2 g)$, respectively. Let $k=\mathbf{Q}$ and take for $\Gamma$ the arithmetic subgroup $\mathrm{SL}(n, \mathbf{Z})$ or $\operatorname{Sp}(2 g, \mathbf{Z})$ of $\mathbf{G}$, respectively. The Weyl groups are, respectively, the symmetric group on $n$ letters and the group of signed permutations on $g$ letters. In those cases (where $k_{\mathbf{G}}=\mathbf{Q}$ and $\Pi(\mathbf{G})=W(\mathbf{G})$ ), Theorem 1.1 was proved in $[\mathrm{K}$, Th. 7.12] when $k=\mathbf{Q}$.

(2) For an example with $\Pi(\mathbf{G}) \neq W(\mathbf{G})$, let us take for $\mathbf{G}$ a non-split form of the special orthogonal group $\mathrm{SO}(4)$ over $\mathbf{Q}$. Say, the group corresponds to the positive isometries of the four-dimensional space endowed with the nondegenerate indefinite quadratic form $Q\left(x_{1}, \ldots, x_{4}\right)=$ $x_{1}^{2}+x_{2}^{2}-x_{3}^{2}-x_{4}^{2}$. Of course $\mathbf{G}$ is split over $\mathbf{Q}(i)$.

The Weyl group of $\mathrm{SO}(4)$ is isomorphic to the Klein four group. Indeed this group corresponds to the Weyl group of the root system of type $D_{2}$. However, a "generic" $g \in \mathrm{SO}(4, \mathbf{Z})$ should have 
a characteristic polynomial whose splitting field $k_{g}$ over $\mathbf{Q}$ has Galois group sitting in the exact sequence

$$
1 \rightarrow W(\mathrm{SO}(4)) \rightarrow \operatorname{Gal}\left(k_{g} / \mathbf{Q}\right) \rightarrow \operatorname{Gal}(\mathbf{Q}(i) / \mathbf{Q}) \rightarrow 1
$$

Therefore the "right" maximal Galois group is an extension of $\mathbf{Z} / 2 \mathbf{Z}$ by $W(\mathrm{SO}(4))$, and it is in fact the Weyl group of the root system of type $C_{2}$.

(3) Parts (ii) and (iv) involve a subtlety that we overlooked in the first version of this paper, and which was pointed out by L. Rosenzweig: if $\mathbf{G}$ is reductive, and not semisimple, then in general we can not claim that the convergence in (ii) occurs exponentially fast (in contrast with (iii)). For instance, consider $k=\mathbf{Q}$, and take a hyperbolic element $g_{0}$ in $\operatorname{SL}(2, \mathbf{Z})$. Let $\mathbf{G}$ be the Zariskiclosure of the infinite cyclic subgroup $g_{0}^{\mathbf{Z}}$ generated by $g_{0}$, so that $\mathbf{G}$ is a non-split torus. Take also $\Gamma=g_{0}^{\mathbf{Z}} \subset \mathbf{G}(\mathbf{Q})$ and $S=\left\{g_{0}, 1, g_{0}^{-1}\right\}$. Then for $w=\left(s_{1}, \ldots, s_{n}\right) \in S^{n}, k_{w}$ can be either the quadratic field generated by the eigenvalues of $g_{0}$, or $\mathbf{Q}$ itself, the second case happening exactly when $s_{1} \cdots s_{n}=1$. But if $s_{i}=g_{0}^{m_{i}}$ with $m_{i} \in\{-1,0,1\}$, the condition becomes $m_{1}+\cdots+m_{n}=0$, which occurs with probability approximately $n^{-1 / 2}$ (by the Stirling formula).

In the semisimple case our theorem provides exponential decay, in terms of the "length" of the random walk, of the probability that the Galois group is "small". In the general reductive case, one can very likely also derive a general quantitative bound, though only with polynomial decay, and it should be possible to characterize those groups $\mathbf{G}$ for which one can recover exponential decay.

Remark 1.3. (1) There are some interesting connections between our results and ideas introduced by Prasad and Rapinchuk [PrR, $\S 3]$ to study the relation of "weak commensurability" in arithmetic groups.

(2) There are other ways to try to describe "random" elements in an arithmetic group; we comment on these in Section 7, and indicate in particular some interesting natural questions which arise from the probabilistic construction we have chosen.

The plan of the paper is as follows. In Section 2 we analyze, in general, splitting fields of the type considered and relate them with splitting fields of maximal tori in $\mathbf{G}$, which are more intrinsic; this leads to a very general form of the a priori inclusion which is part (i) of the theorem above. Section 3 is also of a preliminary nature and discusses fairly standard facts on reduction of arithmetic groups modulo primes. In Section 4, we show that the general construction, in this case, is closely related to earlier results of Fulman [F] and Carter [Ca2], and we prove an equidistribution statement that will be useful for setting up the sieve (and which is of independent interest). In Section 5, we prove a general sieve result for arithmetic subgroups of semisimple groups - again, a result of independent interest, where other deep ingredients come into play, coming both from algebra (strong approximation results for arithmetic groups) and from harmonic analysis (Property $(\tau)$ ). Finally, in Section 6, we combine the algebraic information with the sieve result and some additional reduction steps in order to obtain the general conclusion. In Section 7, we compare our approach with two other natural ways of quantifying the idea that "random" elements have the Weyl groups as Galois group.

Notation. As usual, $|X|$ denotes the cardinality of a set. For any integer $n \geqslant 1, \mathfrak{S}_{n}$ is the group of permutations on $n$ letters. For any group $G$, we denote by $G^{\sharp}$ the set of conjugacy classes of $G$. We denote by $\mathbf{F}_{q}$ a field with $q$ elements. "Connected" will mean "geometrically connected" for all algebraic groups considered. By the type of a connected reductive algebraic group $\mathbf{G}$ defined over a field $k$ (or a subring of $k$ ), we mean the isomorphism type of its root datum over an algebraic closure of $k$ (see, e.g., [Sp, §9.4]).

By the Galois group of a polynomial, we mean the Galois group of its splitting field. For a number field $k$, we denote by $\mathbf{Z}_{k}$ the ring of integers, and for $\mathfrak{p}$ a prime ideal of $\mathbf{Z}_{k}$, we write $\mathbf{F}_{\mathfrak{p}}$ for the residue field $\mathbf{Z}_{k} / \mathfrak{p} \mathbf{Z}_{k}$. 
For a scheme $X$ defined over a ring $A$ and a ring homomorphism $A \rightarrow B$, we will denote the base extension $X \times_{\operatorname{Spec} A} \operatorname{Spec} B$ by $X_{B}$.

Acknowledgements. Thanks to the referee for a thorough reading, and thanks especially to L. Rosenzweig for both interesting discussions related to this topic and for pointing out a serious mistake in an earlier version.

\section{Splitting Fields of tori AND ElEments of ALGEBRAiC GROUPS}

In this section, we consider the Galois theory of splitting fields of tori and elements in linear algebraic groups. Throughout, let $\mathbf{G}$ be a connected linear algebraic group defined over a perfect field $k$.

2.1. Tori. An algebraic group $\mathbf{T}$ over $k$ is a torus if $\mathbf{T}_{\bar{k}}$ is isomorphic to $\mathbf{G}_{\bar{k}}^{r}$ for an integer $r \geqslant 0$.

Fix a torus $\mathbf{T}$ defined over $k$. We say that $\mathbf{T}$ is split if it is isomorphic over $k$ to $\mathbf{G}_{m}^{r}$. Denote by $X(\mathbf{T})$ the group of characters $\alpha: \mathbf{T}_{\bar{k}} \rightarrow \mathbf{G}_{m, \bar{k}}$, which is a free abelian group of rank equal to the dimension of $\mathbf{T}$. There is a natural action of $\operatorname{Gal}(\bar{k} / k)$ on $X(\mathbf{T})$ given by $\sigma(\chi(t))={ }^{\sigma} \chi(\sigma(t))$ for $\sigma \in \operatorname{Gal}(\bar{k} / k), \chi \in X(\mathbf{T})$ and $t \in \mathbf{T}(\bar{k})$. Let $k_{\mathbf{T}} \subseteq \bar{k}$ be the minimal extension of $k$ for which $\operatorname{Gal}\left(\bar{k} / k_{\mathbf{T}}\right)$ acts trivially on $X(\mathbf{T})$; it is a finite Galois extension of $k$ that we call the splitting field of $\mathbf{T}$. The field $k_{\mathbf{T}}$ is also the minimal extension $K \subseteq \bar{k}$ of $k$ for which $\mathbf{T}_{K}$ is split.

Let $\varphi_{\mathbf{T}}: \operatorname{Gal}(\bar{k} / k) \rightarrow \operatorname{Aut}(X(\mathbf{T}))$ be the representation describing the Galois action on $X(\mathbf{T})$; we have $\varphi_{\mathbf{T}}(\sigma) \chi={ }^{\sigma} \chi$ for all $\sigma \in \operatorname{Gal}(\bar{k} / k)$ and $\chi \in X(\mathbf{T})$. It factors through an injective homomorphism $\operatorname{Gal}\left(k_{\mathbf{T}} / k\right) \hookrightarrow \operatorname{Aut}(X(\mathbf{T}))$.

2.2. Maximal tori. Assume that $\mathbf{G}$ is reductive. Let $\mathbf{T}$ be a maximal torus of $\mathbf{G}$, defined over $k$ (we always consider maximal tori defined over the base field).

In this section, we shall describe a finite subgroup $\Pi(\mathbf{G})$ of $\operatorname{Aut}(X(\mathbf{T}))$ that contains the image of $\varphi_{\mathbf{T}}$ and whose isomorphism class depends only on $\mathbf{G}$.

Let $Z_{\mathbf{G}}(\mathbf{T})$ and $N_{\mathbf{G}}(\mathbf{T})$ be the centralizer and normalizer, respectively, of $\mathbf{T}$ in $\mathbf{G}$. The Weyl group of $\mathbf{G}$ with respect to $\mathbf{T}$, denoted $W(\mathbf{G}, \mathbf{T})$, is defined to be the $\bar{k}$-valued points of $N_{\mathbf{G}}(\mathbf{T}) / Z_{\mathbf{G}}(\mathbf{T})$. The group $W(\mathbf{G}, \mathbf{T})$ is finite.

Conjugation induces an action of $W(\mathbf{G}, \mathbf{T})$ on $\mathbf{T}$; for $w \in W(\mathbf{G}, \mathbf{T})$ represented by an element $n \in N_{\mathbf{G}}(\mathbf{T})(\bar{k})$, we have $w \cdot t:=n t n^{-1}$. This action is faithful since $Z_{\mathbf{G}}(\mathbf{T})=\mathbf{T}$ [Bo, 13.17 Corollary 2]. The Weyl group $W(\mathbf{G}, \mathbf{T})$ also acts faithfully on $X(\mathbf{T})$; for $\chi \in X(\mathbf{T}), w \cdot \chi$ is the character of $\mathbf{T}$ defined by $t \mapsto \chi\left(n^{-1} t n\right)$. Using this last action, we may identify $W(\mathbf{G}, \mathbf{T})$ with a subgroup of $\operatorname{Aut}(X(\mathbf{T}))$.

We define $\Pi(\mathbf{G}, \mathbf{T})$ to be the subgroup of $\operatorname{Aut}(X(\mathbf{T}))$ generated by $W(\mathbf{G}, \mathbf{T})$ and $\varphi_{\mathbf{T}}(\operatorname{Gal}(\bar{k} / k))$. Trivially, we have $\varphi_{\mathbf{T}}(\operatorname{Gal}(\bar{k} / k)) \subseteq \Pi(\mathbf{G}, \mathbf{T})$, so we may rewrite our representation as

$$
\varphi_{\mathbf{T}}: \operatorname{Gal}(\bar{k} / k) \rightarrow \Pi(\mathbf{G}, \mathbf{T}) .
$$

We will now show that the group $\Pi(\mathbf{G}, \mathbf{T})$, up to isomorphism, is independent of $\mathbf{T}$.

Let $\mathbf{T}_{0}$ be a fixed maximal torus of $\mathbf{G}$ defined over $k$. Since all maximal tori of $\mathbf{G}$ are conjugate over $\bar{k}$, there is an element $x \in \mathbf{G}(\bar{k})$ such that $\mathbf{T}_{\bar{k}}=x \mathbf{T}_{0, \bar{k}} x^{-1}$. This gives isomorphisms $f: \mathbf{T}_{\bar{k}} \stackrel{\sim}{\rightarrow}$ $\mathbf{T}_{0, \bar{k}}, t \mapsto x^{-1} t x$ and $F: X(\mathbf{T}) \stackrel{\sim}{\rightarrow} X\left(\mathbf{T}_{0}\right), \chi \mapsto \chi \circ f^{-1}$.

Proposition 2.1. With notation as above, the following hold:

(i) The Weyl group $W(\mathbf{G}, \mathbf{T})$ is a normal subgroup of $\Pi(\mathbf{G}, \mathbf{T})$.

(ii) The map

$$
\operatorname{Aut}(X(\mathbf{T})) \stackrel{\sim}{\rightarrow} \operatorname{Aut}\left(X\left(\mathbf{T}_{0}\right)\right) \quad \gamma \mapsto F \circ \gamma \circ F^{-1}
$$


is an isomorphism which induces isomorphisms

$$
\Pi(\mathbf{G}, \mathbf{T}) \stackrel{\sim}{\rightarrow} \Pi\left(\mathbf{G}, \mathbf{T}_{0}\right), \quad W(\mathbf{G}, \mathbf{T}) \stackrel{\sim}{\rightarrow} W\left(\mathbf{G}, \mathbf{T}_{0}\right) .
$$

A different choice of $x$ gives the same isomorphisms up to composition by an inner automorphism arising from an element of the Weyl group.

(iii) Take $\sigma \in \operatorname{Gal}(\bar{k} / k)$ and let $w_{\sigma}$ be the element of $W(\mathbf{G}, \mathbf{T})$ represented by $x^{-1} \sigma(x) \in$ $N_{\mathbf{G}}(\mathbf{T})(\bar{k})$. Then

$$
F \circ \varphi_{\mathbf{T}}(\sigma) \circ F^{-1}=w_{\sigma} \circ \varphi_{\mathbf{T}_{0}}(\sigma) .
$$

(iv) If $K \subseteq \bar{k}$ is an extension of $k$ for which $\mathbf{G}_{K}$ is split, then $\varphi_{\mathbf{T}}(\operatorname{Gal}(\bar{k} / K)) \subseteq W(\mathbf{G}, \mathbf{T})$.

Proof. (i) For $\sigma \in \operatorname{Gal}(\bar{k} / k)$ and $w \in W(\mathbf{G}, \mathbf{T})$, we need to show that $\varphi_{\mathbf{T}}(\sigma) \circ w \circ \varphi_{\mathbf{T}}(\sigma)^{-1}$ belongs to $W(\mathbf{G}, \mathbf{T})$. For a character $\chi \in X(\mathbf{T})$, we have

$$
\left(\varphi_{\mathbf{T}}(\sigma) \circ w \circ \varphi_{\mathbf{T}}(\sigma)^{-1}\right) \chi={ }^{\sigma}\left(w \cdot \sigma^{-1} \chi\right)=\sigma(w) \cdot \chi
$$

where we are using the natural Galois action on the Weyl group. Therefore, $\varphi_{\mathbf{T}}(\sigma) \circ w \circ \varphi_{\mathbf{T}}(\sigma)^{-1}=$ $\sigma(w)$ which does indeed belong to $W(\mathbf{G}, \mathbf{T})$.

(ii) The isomorphism of Weyl groups is easy to check; if $w \in W(\mathbf{G}, \mathbf{T})$ has representative $n \in$ $N_{\mathbf{G}}(\mathbf{T})(\bar{k})$, then $F \circ w \circ F^{-1}$ belongs to $W\left(\mathbf{G}, \mathbf{T}_{0}\right)$ with representative $x^{-1} n x$. To verify that we have an isomorphism $\Pi(\mathbf{G}, \mathbf{T}) \stackrel{\sim}{\rightarrow} \Pi\left(\mathbf{G}, \mathbf{T}_{0}\right)$, it suffices to show that $F \circ \varphi_{\mathbf{T}}(\sigma) \circ F^{-1}$ belongs to $\Pi\left(\mathbf{G}, \mathbf{T}_{0}\right)$ for all $\sigma \in \operatorname{Gal}(\bar{k} / k)$. For $\chi \in X\left(\mathbf{T}_{0}\right)$,

$$
\left(F \circ \varphi_{\mathbf{T}}(\sigma) \circ F^{-1}\right) \chi={ }^{\sigma}(\chi \circ f) \circ f^{-1}={ }^{\sigma} \chi \circ\left({ }^{\sigma} f \circ f^{-1}\right)={ }^{\sigma} \chi \circ\left(f \circ{ }^{\sigma} f^{-1}\right)^{-1} .
$$

The automorphism $f \circ{ }^{\sigma} f^{-1}$ of $\mathbf{T}_{\bar{k}}$ maps an element $t \in \mathbf{T}(\bar{k})$ to $x^{-1} \sigma(x) t\left(x^{-1} \sigma(x)\right)^{-1}$ which equals $w_{\sigma} \cdot t$ where $w_{\sigma}$ is the element of $W(\mathbf{G}, \mathbf{T})$ represented by $x^{-1} \sigma(x) \in N_{\mathbf{G}}(\mathbf{T})(\bar{k})$ (indeed, since $\mathbf{T}$ and $\mathbf{T}_{0}$ are both defined over $k$, the element $x^{-1} \sigma(x)$ normalizes $\left.\mathbf{T}\right)$. From (2.1), we deduce that $F \circ \varphi_{\mathbf{T}}(\sigma) \circ F^{-1}=w_{\sigma} \circ \varphi_{\mathbf{T}_{0}}(\sigma)$ which certainly belongs to $\Pi\left(\mathbf{G}, \mathbf{T}_{0}\right)$. We have also proved (iii). For (iv), we may assume that $\mathbf{T}_{0}$ was chosen such that $k_{\mathbf{T}_{0}} \subseteq K$. For $\sigma \in \operatorname{Gal}(\bar{k} / K)$, part (iii) implies that $\varphi_{\mathbf{T}}(\sigma)=F^{-1} \circ w_{\sigma} \circ F$ which is an element of $W(\mathbf{G}, \mathbf{T})$ by (ii).

The groups $W(\mathbf{G}, \mathbf{T})$ and $\Pi(\mathbf{G}, \mathbf{T})$ are, up to isomorphism, independent of $\mathbf{T}$ (by Proposition 2.1(ii)). We shall denote the abstract groups simply by $W(\mathbf{G})$ and $\Pi(\mathbf{G})$, respectively, when the choice of torus is unimportant. The isomorphisms $\Pi(\mathbf{G}, \mathbf{T}) \stackrel{\sim}{\rightarrow} \Pi\left(\mathbf{G}, \mathbf{T}_{0}\right)$ and $W(\mathbf{G}, \mathbf{T}) \stackrel{\sim}{\rightarrow}$ $W\left(\mathbf{G}, \mathbf{T}_{0}\right)$ of Proposition 2.1 are unique up to composition with an inner automorphism by an element of the Weyl group; hence they induce canonical bijections $W(\mathbf{G}, \mathbf{T})^{\sharp}=W\left(\mathbf{G}, \mathbf{T}_{0}\right)^{\sharp}$ and $\Pi(\mathbf{G}, \mathbf{T})^{\sharp}=\Pi\left(\mathbf{G}, \mathbf{T}_{0}\right)^{\sharp}$ of conjugacy classes. The set $W(\mathbf{G})^{\sharp}$ and $\Pi(\mathbf{G})^{\sharp}$ are thus completely unambiguous.

We define the splitting field of $\mathbf{G}$ to be the field $k_{\mathbf{G}}:=\bigcap_{\mathbf{T}} k_{\mathbf{T}}$ where the intersection is over all maximal tori $\mathbf{T}$ of $\mathbf{G}$. In other words, $k_{\mathbf{G}}$ is the largest extension of $k$ that is contained in any $K \subseteq \bar{k}$ for which $\mathbf{G}_{K}$ is split.

Lemma 2.2. For every maximal torus $\mathbf{T}$ of $\mathbf{G}$, we have $\varphi_{\mathbf{T}}\left(\operatorname{Gal}\left(\bar{k} / k_{\mathbf{G}}\right)\right) \subseteq W(\mathbf{G})$. In particular, $\operatorname{Gal}\left(k_{\mathbf{T}} / k_{\mathbf{G}}\right)$ is isomorphic to a subgroup of $W(\mathbf{G})$.

Proof. Let $K \subseteq \bar{k}$ be the minimal extension of $k$ for which $\varphi_{\mathbf{T}}(\operatorname{Gal}(\bar{k} / K)) \subseteq W(\mathbf{G}, \mathbf{T})$ (this is well-defined since $W(\mathbf{G})$ is a normal subgroup of $\Pi(\mathbf{G})$ ). For a maximal torus $\mathbf{T}_{0}$ of $\mathbf{G}$, Proposition 2.1(iv) implies that $K \subseteq k_{\mathbf{T}_{0}}$. Since $\mathbf{T}_{0}$ was arbitrary, we deduce that $K \subseteq k_{\mathbf{G}}$. 
2.3. Galois groups for elements. Choose a faithful representation $\rho$ : $\mathbf{G} \hookrightarrow \operatorname{GL}(m)$ defined over $k$. For $g \in \mathbf{G}(k)$, we define $k_{g}$ to be the splitting field over $k$ of $\operatorname{det}(T-\rho(g))$.

Recall that each $g \in \mathbf{G}(k)$ equals $g_{s} g_{u}$ for unique commuting elements $g_{s}, g_{u} \in \mathbf{G}(k)$ where $g_{s}$ is semisimple and $g_{u}$ is unipotent. Since $\operatorname{det}(T-\rho(g))=\operatorname{det}\left(T-\rho(g)_{s}\right)=\operatorname{det}\left(T-\rho\left(g_{s}\right)\right)$, we have $k_{g}=k_{g_{s}}$. The unipotent radical $R_{u}(\mathbf{G})$ of $\mathbf{G}$ is the maximal connected unipotent normal subgroup of $\mathbf{G}$. The quotient $\mathbf{G} / R_{u}(\mathbf{G})$ is reductive and defined over $k$.

\section{Lemma 2.3.}

(i) The field $k_{g}$ does not depend on the choice of $\rho$.

(ii) Define the reductive group $\mathbf{G}^{\prime}:=\mathbf{G} / R_{u}(\mathbf{G})$ and let $\pi: \mathbf{G} \rightarrow \mathbf{G}^{\prime}$ be the quotient homomorphism. Then $k_{g}=k_{\pi(g)}$ for all $g \in \mathbf{G}(k)$.

Proof. Let $\mathbf{D}$ be the algebraic subgroup of $\mathbf{G}$ generated by $g_{s}$. The group $\mathbf{D}$ is diagonalizable, i.e., $\mathbf{D}_{\bar{k}}$ is isomorphic to a subgroup of some torus $\mathbf{G}_{m, \bar{k}}^{r}$. Let $K$ be the splitting field of $\mathbf{D}$, that is, the smallest extension $K \subseteq \bar{k}$ of $k$ for which $\mathbf{D}_{K}$ is isomorphic to a subgroup of a split torus $\mathbf{G}_{m, K}^{r}$. By [Bo, §8.4], we find that $K$ is also the smallest extension of $k$ such that a $\mathrm{GL}(m, K)$-conjugate of $\rho\left(\mathbf{D}_{K}\right)$ is contained in the diagonal subgroup of $\mathrm{GL}(m)$. Equivalently, $K$ is the smallest extension of $k$ for which $\rho\left(g_{s}\right)=\rho(g)_{s}$ is $\mathrm{GL}(m, K)$-conjugate to a diagonal matrix. Therefore, $K=k_{g}$ and part (i) follows since our description of $K$ does not depend on $\rho$.

Let $\mathbf{D}^{\prime}$ be the algebraic subgroup of $\mathbf{G}^{\prime}$ generated by $\pi(g)_{s}=\pi\left(g_{s}\right)$. We have $\mathbf{D} \cap R_{u}(\mathbf{G})=1$ since the only semisimple and unipotent element is 1 . Therefore, $\left.\pi\right|_{\mathbf{D}}: \mathbf{D} \rightarrow \mathbf{D}^{\prime}$ is an isomorphism of algebraic groups. Since $\mathbf{D}$ and $\mathbf{D}^{\prime}$ are isomorphic, we must have $k_{g}=k_{\pi(g)}$.

Recall that a semisimple $g \in \mathbf{G}(k)$ is regular in $\mathbf{G}$ if it is contained in a unique maximal torus; we shall denote this maximal torus by $\mathbf{T}_{g}$. For a semisimple and regular $g \in \mathbf{G}(k)$, we define

$$
\varphi_{g}: \operatorname{Gal}(\bar{k} / k) \rightarrow \Pi(\mathbf{G})
$$

to be the representation denoted by $\varphi_{\mathbf{T}_{g}}$ in the previous section. The representation $\varphi_{g}$ is uniquely defined up to an inner automorphism by an element of $W(\mathbf{G})$.

We will now relate the fields $k_{g}$ to the Galois extensions arising from maximal tori of $\mathbf{G}$.

Lemma 2.4. Assume that $\mathbf{G}$ is reductive.

(i) For all $g \in \mathbf{G}(k), \operatorname{Gal}\left(k_{g} / k\right)$ is isomorphic to a subquotient of $\Pi(\mathbf{G})$ and $\operatorname{Gal}\left(k_{\mathbf{G}} k_{g} / k_{\mathbf{G}}\right)$ is isomorphic to a subquotient of $W(\mathbf{G})$.

(ii) For $g \in \mathbf{G}(k)$, the field $k_{g}$ is the extension of $k$ generated by the set $\left\{\chi\left(g_{s}\right): \chi \in X(\mathbf{T})\right\}$ where $\mathbf{T}$ is a maximal torus of $\mathbf{G}$ containing $g_{s}$.

(iii) There is a closed subvariety $Y \subsetneq \mathbf{G}$ that is stable under conjugation by $\mathbf{G}$ such that if $g \in \mathbf{G}(k)-Y(k)$, then $g$ is semisimple and regular in $\mathbf{G}$ and $k_{g}=k_{\mathbf{T}_{g}}$.

Proof. We start with (ii). Take $g \in \mathbf{G}(k)$. Since $k_{g}=k_{g_{s}}$, we may assume that $g$ is semisimple. Fix a maximal torus $\mathbf{T}$ containing $g$, and let $\Omega \subseteq X(\mathbf{T})$ be the set of weights arising from the representation $\left.\rho\right|_{\mathbf{T}}: \mathbf{T} \hookrightarrow \mathrm{GL}(m)$. There are positive integers $m_{\chi}$ such that

$$
\operatorname{det}(T-\rho(t))=\prod_{\chi \in \Omega}(T-\chi(t))^{m_{\chi}}
$$

for all $t \in \mathbf{T}(\bar{k})$, and in particular, $\{\chi(g): \chi \in \Omega\}$ is the set of $\operatorname{roots}$ of $\operatorname{det}(T-\rho(g))$ in $\bar{k}$. The set $\Omega$ generates the group $X(\mathbf{T})$ since the representation $\left.\rho\right|_{\mathbf{T}}: \mathbf{T} \rightarrow \mathrm{GL}(m)$ is faithful. Therefore, we see that the extension of $k$ generated by $\{\chi(g): \chi \in X(\mathbf{T})\}$ is equal to $k_{g}=k(\{\chi(g): \chi \in \Omega\})$, which completes the proof of (ii).

Now we prove (i). For $\sigma \in \operatorname{Gal}(\bar{k} / k)$, we have

$$
\sigma(\chi(g))=\underset{6}{\sigma} \chi(\sigma))={ }^{\sigma} \chi(g)
$$


for all $\chi \in \Omega$. In particular, $\sigma(\chi(g))=\chi(g)$ for all $\sigma \in \operatorname{Gal}\left(\bar{k} / k_{\mathbf{T}}\right)$. Since $k_{g}$ is generated over $k$ by $\{\chi(g): \chi \in \Omega\}$, we deduce that $k_{\mathbf{T}} \supseteq k_{g}$. Part (i) follows, since in $\S 2.2$ we saw that $\mathrm{Gal}\left(k_{\mathbf{T}} / k\right)$ was isomorphic to a subgroup of $\Pi(\mathbf{G})$ and $\operatorname{Gal}\left(k_{\mathbf{T}} / k_{\mathbf{G}}\right)$ was isomorphic to a subgroup of $W(\mathbf{G})$.

Now for (iii). First of all, there is a closed subvariety $Y_{1} \subseteq \mathbf{G}$ such that $h \in \mathbf{G}(\bar{k})$ does not belong to $Y_{1}(\bar{k})$ if and only if $h$ is semisimple and regular in $\mathbf{G}_{\bar{k}}$, see [St1, 2.14] (the proof is given there only for semisimple groups, but the reductive case follows easily from the semisimple case by considering the morphism from $\mathbf{G}$ to $\mathbf{G} / R_{u}(\mathbf{G})$ ).

Now fix a maximal torus $\mathbf{T}_{0} \subset \mathbf{G}$, and let $\Omega_{0}$ be the set of weights of $\mathbf{T}_{0}$ with respect to $\rho$, as above. The set

$$
V=\left\{t \in \mathbf{T}_{0} \mid \text { the } \chi(t) \text { are distinct for } \chi \in \Omega_{0}\right\}
$$

is an open dense subset of $\mathbf{T}_{0}$. Arguing as in [St1, 2.14], it follows that the set $Y_{2}$ of those $h \in \mathbf{G}$ where $g_{s}$ is conjugate in $\mathbf{G}$ to an element in $\mathbf{T}_{0}-V$ is a proper subvariety of $\mathbf{G}$. Moreover, it is clearly invariant under conjugation.

Now we define the proper closed subvariety $Y=Y_{1} \cup Y_{2}$ of $\mathbf{G}$, which is stable under conjugation, and we claim that (iii) holds. Indeed, let $g \in \mathbf{G}(k)-Y(k)$. Since $g \notin Y_{1}(k)$, it is a regular semisimple element of $\mathbf{G}$. Let $\mathbf{T}_{g}$ be the unique maximal torus containing $g$, $\Omega$ the set of weights with respect to $\mathbf{T}_{g}$. Since $g \notin Y_{2}(k)$ and $\Omega$ is obtained from $\Omega_{0}$ by conjugation, it follows that the values $\chi(g), \chi \in \Omega$, are all distinct.

But now, take any $\sigma \in \operatorname{Gal}\left(\bar{k} / k_{g}\right)$. By $(2.2)$, we have ${ }^{\sigma} \chi(g)=\chi(g)$ for all $\chi \in \Omega$, and therefore we must have in fact ${ }^{\sigma} \chi=\chi$ for all $\chi \in \Omega$. Since $\Omega$ generates the group $X\left(\mathbf{T}_{g}\right)$, we find that $\sigma$ acts trivially on $X\left(\mathbf{T}_{g}\right)$, and since $\sigma$ was an arbitrary element of $\operatorname{Gal}\left(\bar{k} / k_{g}\right)$, we deduce finally that $k_{g} \supseteq k_{\mathbf{T}_{g}}$.

\section{REDUCTIONS OF ARITHMEtiC GROUPS AND TORI OVER FINITE FIELDS}

Let $\mathbf{G}$ be a connected semisimple group defined over a number field $k$. To consider reductions, we will need to choose a model of $\mathbf{G}$. This means that we take a group scheme $\mathcal{G}$ over a ring $\mathbf{Z}_{k}\left[R^{-1}\right]$ whose generic fiber $\mathcal{G}_{k}$ is isomorphic to $\mathbf{G}$, where $R$ is a finite set of maximal ideals of $\mathbf{Z}_{k}$. We identify $\mathbf{G}$ with the generic fiber of $\mathcal{G}$. Any two such models will agree after possibly inverting more primes. From now on, $\mathfrak{p}$ will denote a maximal ideal of $\mathbf{Z}_{k}$. Let $k_{\mathfrak{p}}$ be the completion of $k$ at the prime $\mathfrak{p}$ and let $\mathcal{O}_{\mathfrak{p}}$ be the corresponding valuation ring. The ring $\mathcal{O}_{\mathfrak{p}}$ is a discrete valuation ring with residue field $\mathbf{F}_{\mathfrak{p}}$.

After possibly increasing $R$, we may assume that $\mathcal{G}$ is semisimple and that all of its fibers have the same type. For background on general reductive groups, see [D]; recall that $\mathcal{G}$ is semisimple if it is affine and smooth over $\mathbf{Z}_{k}\left[R^{-1}\right]$ and if the generic fiber $\mathcal{G}_{k}$ and special fibers $\mathcal{G}_{\mathbf{F}_{\mathfrak{p}}}(\mathfrak{p} \notin R)$ are semisimple in the usual sense.

Choose a maximal torus $\mathcal{T}_{0}$ of $\mathcal{G}$. Let $\mathcal{P}$ be the set of maximal ideals $\mathfrak{p} \notin R$ of $\mathbf{Z}_{k}$ such that the tori $\mathcal{T}_{0, k_{\mathfrak{p}}}$ and $\mathcal{T}_{0, \mathbf{F}_{\mathfrak{p}}}$ are both split.

Lemma 3.1. Let $\mathcal{N}_{0}$ be the normalizer of $\mathcal{T}_{0}$ in $\mathcal{G}$. For each $\mathfrak{p} \in \mathcal{P}$, there is a unique bijection

$$
W\left(\mathcal{G}_{k_{\mathfrak{p}}}\right)^{\sharp} \leftrightarrow W\left(\mathcal{G}_{\mathbf{F}_{\mathfrak{p}}}\right)^{\sharp}
$$

such that for $n \in \mathcal{N}_{0}\left(\mathcal{O}_{\mathfrak{p}}\right)$ the image of $n$ in $W\left(\mathcal{G}_{k_{\mathfrak{p}}}, \mathcal{T}_{0, k_{\mathfrak{p}}}\right)^{\sharp}$ and $W\left(\mathcal{G}_{\mathbf{F}_{\mathfrak{p}}}, \mathcal{T}_{0, \mathbf{F}_{\mathfrak{p}}}\right)^{\sharp}$ correspond.

Proof. The homomorphism

$$
\mathcal{N}_{0}\left(\mathcal{O}_{\mathfrak{p}}\right) / \mathcal{T}_{0}\left(\mathcal{O}_{\mathfrak{p}}\right) \hookrightarrow \mathcal{N}_{0}\left(k_{\mathfrak{p}}\right) / \mathcal{T}_{0}\left(k_{\mathfrak{p}}\right)=W\left(\mathcal{G}_{k_{\mathfrak{p}}}, \mathcal{T}_{0, k_{\mathfrak{p}}}\right)
$$

is injective; the identification with the Weyl group uses that $\mathcal{T}_{0, k_{\mathfrak{p}}}$ is split. The normalizer $\mathcal{N}_{0}$ is a closed and smooth subscheme of $\mathcal{G}$ (for smoothness, cf. [SGA3, XXII Corollaire 5.3.10]). The 
homomorphisms $\mathcal{N}_{0}\left(\mathcal{O}_{\mathfrak{p}}\right) \rightarrow \mathcal{N}_{0}\left(\mathbf{F}_{\mathfrak{p}}\right)$ and $\mathcal{T}_{0}\left(\mathcal{O}_{\mathfrak{p}}\right) \rightarrow \mathcal{T}_{0}\left(\mathbf{F}_{\mathfrak{p}}\right)$ are surjective by Hensel's lemma. We thus have a surjective homomorphism

$$
\mathcal{N}_{0}\left(\mathcal{O}_{\mathfrak{p}}\right) / \mathcal{T}_{0}\left(\mathcal{O}_{\mathfrak{p}}\right) \rightarrow \mathcal{N}_{0}\left(\mathbf{F}_{\mathfrak{p}}\right) / \mathcal{T}_{0, \mathbf{F}_{\mathfrak{p}}}\left(\mathbf{F}_{\mathfrak{p}}\right)=W\left(\mathcal{G}_{\mathbf{F}_{\mathfrak{p}}}, \mathcal{T}_{0, \mathbf{F}_{\mathfrak{p}}}\right)
$$

where the equality uses that $\mathcal{T}_{0, \mathbf{F}_{\mathfrak{p}}}$ is split. The Weyl groups $W\left(\mathcal{G}_{k_{\mathfrak{p}}}, \mathcal{T}_{0, k_{\mathfrak{p}}}\right)$ and $W\left(\mathcal{G}_{\mathbf{F}_{\mathfrak{p}}}, \mathcal{T}_{0, \mathbf{F}_{\mathfrak{p}}}\right)$ are isomorphic since $\mathcal{G}_{k_{\mathfrak{p}}}$ and $\mathcal{G}_{\mathbf{F}_{\mathfrak{p}}}$ have the same type. Since (3.1) and (3.2) are injective and surjective, respectively, we deduce that they are both isomorphisms. By combining them, we get an isomorphism

$$
W\left(\mathcal{G}_{k_{\mathfrak{p}}}, \mathcal{T}_{0, k_{\mathfrak{p}}}\right) \stackrel{\sim}{\rightarrow} W\left(\mathcal{G}_{\mathbf{F}_{\mathfrak{p}}}, \mathcal{T}_{0, \mathbf{F}_{\mathfrak{p}}}\right) .
$$

The desired bijection of conjugacy classes is induced from this isomorphism. The uniqueness is a consequence of the surjectivity of (3.2).

Fix a maximal ideal $\mathfrak{p} \in \mathcal{P}$ and choose an embedding $\iota: \bar{k} \hookrightarrow \overline{k_{\mathfrak{p}}}$ that is the identity on $k$. Using $\iota$, we can make an identification $W\left(\mathbf{G}, \mathcal{T}_{0, k}\right)=W\left(\mathcal{G}_{k_{\mathfrak{p}}}, \mathcal{T}_{0, k_{\mathfrak{p}}}\right)$. Combining with the map of Lemma 3.1, we obtain a bijection

$$
W(\mathbf{G})^{\sharp} \leftrightarrow W\left(\mathcal{G}_{\mathbf{F}_{\mathfrak{p}}}\right)^{\sharp}
$$

that we will also use as an identification. For an element $g \in \mathcal{G}\left(\mathbf{F}_{\mathfrak{p}}\right)$ that is semisimple and regular in $\mathcal{G}_{\mathbf{F}_{\mathfrak{p}}}$, we have a homomorphism

$$
\varphi_{g}: \operatorname{Gal}\left(\overline{\mathbf{F}}_{\mathfrak{p}} / \mathbf{F}_{\mathfrak{p}}\right) \rightarrow W(\mathbf{G})
$$

by using that $\mathcal{T}_{0, \mathbf{F}_{\mathfrak{p}}}$ is split. Let Frob $\mathfrak{p}$ be the Frobenius automorphism $x \mapsto x^{N(\mathfrak{p})}$ of $\overline{\mathbf{F}}_{\mathfrak{p}}$ where $N(\mathfrak{p})$ is the cardinality of $\mathbf{F}_{\mathfrak{p}}$. The representation $\varphi_{g}$ (up to inner automorphism) is determined by the conjugacy class $\varphi_{g}\left(\right.$ Frob $\left._{\mathfrak{p}}\right)$ of $W(\mathbf{G})$.

The following crucial proposition shows that the local and global images of Frobenius automorphisms coincide.

Proposition 3.2 (Local and global Frobenius). Let $\mathfrak{p} \in \mathcal{P}$ be a prime ideal. Let $g \in \mathcal{G}\left(\mathbf{Z}_{k}\left[R^{-1}\right]\right)$ be an element such that $g$ is semisimple and regular in $\mathbf{G}=\mathcal{G}_{k}$ and $\bar{g}:=g \bmod \mathfrak{p} \in \mathcal{G}\left(\mathbf{F}_{\mathfrak{p}}\right)$ is semisimple and regular in $\mathcal{G}_{\mathbf{F}_{\mathfrak{p}}}$. Let $C$ be the conjugacy class of $W(\mathbf{G})$ containing $\varphi_{\bar{g}}\left(\right.$ Frob $\left._{\mathfrak{p}}\right)$. Then the representation $\varphi_{g}$ is unramified at $\mathfrak{p}$, and if $\sigma_{\mathfrak{p}} \in \operatorname{Gal}(\bar{k} / k)$ denotes a Frobenius element at $\mathfrak{p}$, we have

$$
\varphi_{g}\left(\sigma_{\mathfrak{p}}\right) \in C
$$

This is intuitively very natural, but the proof requires some care in our generality, in particular to show that the representation is unramified. In previous works, this issue did not come up, since one could explicitly control factorizations of the reduction of the characteristic polynomial to ensure it was squarefree in suitable conditions.

Because of this proposition, we will, from now on, also denote by Frob $\mathfrak{p}$ any representative of the Frobenius automorphism in $\operatorname{Gal}(\bar{k} / k)$.

Proof. Let $k_{\mathfrak{p}}^{\text {un }}$ denote the maximal unramified extension of $k_{\mathfrak{p}}$ in an algebraic closure $\overline{k_{\mathfrak{p}}}$. Let $\mathcal{O}_{\mathfrak{p}}^{\text {un }}$ be the valuation ring of $k_{\mathfrak{p}}^{u n}$; its residue field is $\overline{\mathbf{F}}_{\mathfrak{p}}$. We have an isomorphism

$$
\operatorname{Gal}\left(k_{\mathfrak{p}}^{u n} / k_{\mathfrak{p}}\right) \stackrel{\sim}{\rightarrow} \operatorname{Gal}\left(\overline{\mathbf{F}}_{\mathfrak{p}} / \mathbf{F}_{\mathfrak{p}}\right),
$$

which allows us to view Frob $b_{\mathfrak{p}}$ as an automorphism of $k_{\mathfrak{p}}^{\text {un }}$. We will also denote by Frob $b_{\mathfrak{p}}$ any extension of the Frobenius automorphism to the field $\overline{k_{\mathfrak{p}}}$

We now need to compare maximal tori in $\mathcal{G}_{\mathcal{O}_{\mathfrak{p}}}$ and $\mathcal{G}_{\mathbf{F}_{\mathfrak{p}}}$. First, we have the tori $\mathcal{T}_{0, \mathcal{O}_{\mathfrak{p}}}$ (which we will still denote $\mathcal{T}_{0}$ for simplicity) and its reduction $\mathcal{T}_{0, \mathbf{F}_{\mathfrak{p}}}$ modulo $\mathfrak{p}$. Further, because we assume that $g$ (as element of $\mathcal{G}\left(k_{\mathfrak{p}}\right.$ ), i.e., of the generic fiber of $\mathcal{G}_{\mathcal{O}_{\mathfrak{p}}}$ ) and $\bar{g}$ (as section of $\mathcal{G}_{\mathcal{O}_{\mathfrak{p}}}$ over the special 
fiber) are regular semisimple, there exists a unique maximal torus $\mathcal{T}$ of $\mathcal{G}_{\mathcal{O}_{\mathfrak{p}}}$ containing $g$ (this is a special case of [SGA3, XIII, Cor. 3.2]).

The transporter $\operatorname{Transp}_{\mathcal{G}}\left(\mathcal{T}_{0}, \mathcal{T}\right)$, defined as an $\mathcal{O}_{\mathfrak{p}}$-scheme by

$$
\operatorname{Transp}_{\mathcal{G}}\left(\mathcal{T}_{0}, \mathcal{T}\right)(A)=\left\{g \in \mathcal{G}(A): g \mathcal{T}_{0, A} g^{-1}=\mathcal{T}_{A}\right\}
$$

is a closed and smooth group scheme in $\mathcal{G}_{\mathcal{O}_{\mathfrak{p}}}$ (for smoothness, cf. [SGA3, XXII Corollaire 5.3.10]).

Now we can choose an element $\bar{x} \in \operatorname{Transp}_{\mathcal{G}}\left(\mathcal{T}_{0}, \mathcal{T}\right)\left(\overline{\mathbf{F}}_{\mathfrak{p}}\right)$. Since $\operatorname{Transp} \mathrm{G}_{\mathcal{G}}\left(\mathcal{T}_{0}, \mathcal{T}\right)$ is smooth and $\mathcal{O}_{\mathfrak{p}}^{\text {un }}$ is a Henselian ring, there is an $x \in \operatorname{Transp}_{\mathcal{G}}\left(\mathcal{T}_{0}, \mathcal{T}\right)\left(\mathcal{O}_{\mathfrak{p}}^{\text {un }}\right)$ which lifts $\bar{x}$.

Finally, by Proposition 2.1(iii), the conjugacy class of $\varphi_{\mathcal{T}_{k_{\mathfrak{p}}}}\left(\right.$ Frob $\left._{\mathfrak{p}}\right)$ in $W\left(\mathcal{G}_{k_{\mathfrak{p}}}\right)^{\sharp}=W\left(\mathcal{G}_{k_{\mathfrak{p}}}, \mathcal{T}_{0, k_{\mathfrak{p}}}\right)^{\sharp}$ is represented by $x^{-1} \operatorname{Frob}_{\mathfrak{p}}(x) \in N_{\mathcal{G}_{k \mathfrak{p}}}\left(\mathcal{T}_{0, k_{\mathfrak{p}}}\right)$. Similarly, the conjugacy class of $\varphi_{\bar{g}}\left(\mathrm{Frob}_{\mathfrak{p}}\right)=$

$\varphi_{\mathcal{T}_{\mathfrak{p}}}\left(\right.$ Frob $\left._{\mathfrak{p}}\right)$ in $W\left(\mathcal{G}_{\mathbf{F}_{\mathfrak{p}}}\right)^{\sharp}=W\left(\mathcal{G}_{\mathbf{F}_{\mathfrak{p}}}, \mathcal{T}_{0, \mathbf{F}_{\mathfrak{p}}}\right)^{\sharp}$ is represented by $\bar{x}^{-1}$ Frob $_{\mathfrak{p}}(\bar{x}) \in N_{\mathcal{G}_{\mathbf{F}_{\mathfrak{p}}}}\left(\mathcal{T}_{0, \mathbf{F}_{\mathfrak{p}}}\right)$. These conjugacy correspond under the bijection of Lemma 3.1. In particular, $\varphi_{\mathcal{T}_{k \mathfrak{p}}}\left(\right.$ Frob $\left._{\mathfrak{p}}\right)$ does not depend on the choice of extension Frob $b_{\mathfrak{p}}$ i.e., $\varphi_{\mathcal{T}_{k_{\mathfrak{p}}}}$ is unramified at $\mathfrak{p}$. The choice of $\iota$ gives an inclusion $\iota^{*}: \operatorname{Gal}\left(\overline{k_{\mathfrak{p}}} / k_{\mathfrak{p}}\right) \hookrightarrow \operatorname{Gal}(\bar{k} / k)$. The representation $\varphi_{\mathcal{T}_{k_{\mathfrak{p}}}}$ is equal to $\iota^{*}$ composed with $\varphi_{g}$. The proposition now follows immediately.

To show that $\varphi_{g}$ is often surjective, we will exploit the well-known lemma of Jordan according to which, in a finite group, no proper subgroup contains elements of all conjugacy classes. Proposition 3.2 will be used to produce conjugacy classes of $W(\mathbf{G})$ that intersect $\varphi_{g}\left(\mathrm{Gal}\left(\bar{k} / k_{\mathbf{G}}\right)\right)$.

\section{Semisimple elements and COnjugacy Classes in the Weyl group over finite fields}

In this section, which can be read independently of the rest of the paper, we consider a finite field $k=\mathbf{F}_{q}$ with $q$ elements and a connected split semisimple group $\mathbf{G}$ defined over $\mathbf{F}_{q}$. In $\S 2$, we defined a homomorphism

$$
\varphi_{\mathbf{T}}: \operatorname{Gal}\left(\overline{\mathbf{F}}_{q} / \mathbf{F}_{q}\right) \rightarrow W(\mathbf{G}, \mathbf{T})
$$

for each maximal torus $\mathbf{T}$ of $\mathbf{G}$ (the image lies in $W(\mathbf{G}, \mathbf{T})$ by Proposition 2.1(iv)). The representation $\varphi_{\mathbf{T}}$ is determined by its value on the Frobenius automorphism $F: x \mapsto x^{q}$, and $\varphi_{\mathbf{T}}(F)$ gives a well-defined conjugacy class in $W(\mathbf{G}, \mathbf{T})^{\sharp}=W(\mathbf{G})^{\sharp}$ that we shall denote by $\theta(\mathbf{T})$.

Now if $g \in \mathbf{G}\left(\mathbf{F}_{q}\right)$ is a semisimple regular element of $\mathbf{G}$, then it is contained in a unique maximal torus $\mathbf{T}_{g}$ of $\mathbf{G}$, and we will study here the map

$$
\theta:\left\{\begin{array}{cl}
\mathbf{G}\left(\mathbf{F}_{q}\right)_{s r} & \rightarrow W(\mathbf{G})^{\sharp} \\
g & \mapsto \theta\left(\mathbf{T}_{g}\right)=\left[\varphi_{\mathbf{T}_{g}}(F)\right]
\end{array}\right.
$$

where $\mathbf{G}\left(\mathbf{F}_{q}\right)_{s r}$ is the set of regular and semisimple elements of $\mathbf{G}\left(\mathbf{F}_{q}\right)$. From Proposition 2.1(iii), it follows that this can be described concretely as follows: we fix a split maximal torus $\mathbf{T}_{0} \subset \mathbf{G}$, and then, given $g \in \mathbf{G}\left(\mathbf{F}_{q}\right)_{s r}$, let $\mathbf{T}$ be the unique maximal torus containing $g$. Take $y \in \mathbf{G}\left(\overline{\mathbf{F}}_{q}\right)$ such that

$$
\mathbf{T}=y \mathbf{T}_{0} y^{-1} .
$$

Then $\theta(g)$ is the class of $y^{-1} F(y)$ in $W\left(\mathbf{G}, \mathbf{T}_{0}\right)^{\sharp}=W(\mathbf{G})^{\sharp}$.

Our goal is to prove that the values of this map are asymptotically equidistributed, with respect to the natural measure on the conjugacy classes of $W(\mathbf{G})$, when $q$ goes to infinity (and the type of $G$ is fixed).

Proposition 4.1. For each $C \in W(\mathbf{G})^{\sharp}$, we have

$$
\frac{\left|\left\{g \in \mathbf{G}\left(\mathbf{F}_{q}\right)_{s r}: \theta(g)=C\right\}\right|}{\left|\mathbf{G}\left(\mathbf{F}_{q}\right)\right|}=\frac{|C|}{|W(\mathbf{G})|}\left(1+O\left(q^{-1}\right)\right)
$$

where the implicit constant depends only on the type of $\mathbf{G}$. 
Remark 4.2. If $\mathbf{G}$ is a simple and simply-connected group, Theorem 1 of [Ca1] describes precisely the number of semisimple conjugacy classes of $\mathbf{G}\left(\mathbf{F}_{q}\right)$ mapping to $C$ under $\theta$ in terms of the geometry of the action of the so-called affine Weyl group on the cocharacter group of a maximal torus of G. A proof of Proposition 4.1 can then be derived fairly easily by a lattice-point counting technique, the well-known formula for the volume of the fundamental domain of the affine Weyl group, and some equidistribution of semisimple conjugacy classes. Our proof is different; it requires less precise information (and works for arbitrary connected semisimple groups), exploiting the fact that we only look for asymptotic information for large $q$.

Remark 4.3. This map has already been considered by Fulman [F] and Carter [Ca2] in the context of finite groups of Lie type. As they remark, it takes a very classical and concrete form when $\mathbf{G}=\mathrm{SL}(m)$. In that case, the Weyl group is the symmetric group $\mathfrak{S}_{m}$, and its conjugacy classes correspond naturally to partitions of the integer $m$. Now, consider an element $g \in \operatorname{SL}\left(m, \mathbf{F}_{q}\right)$ which is regular and has distinct eigenvalues in $\overline{\mathbf{F}}_{q}$; in that case its characteristic polynomial $\operatorname{det}(T-g) \in \mathbf{F}_{q}[T]$ is monic, squarefree and of degree $m$. We may factor it as a product of distinct irreducible factors

$$
\operatorname{det}(T-g)=\pi_{1} \cdots \pi_{k}
$$

and the degrees $d_{i}=\operatorname{deg}\left(\pi_{i}\right)$ form a partition $\lambda$ of $m$ (with as many cycles of length $j$, for $1 \leqslant j \leqslant m$, as there are factors of degree $d_{i}$ equal to $j$ ); then one can check that $\theta(g)$ is the conjugacy class in $\mathfrak{S}_{m}$ corresponding precisely to this partition.

4.1. Proof of Proposition 4.1. We will first need a few lemmas. The notation in this section is the same as before.

Lemma 4.4. The map $\mathbf{T} \mapsto \theta(\mathbf{T})$ defines a bijection between the maximal tori of $\mathbf{G}$ up to conjugation by $\mathbf{G}\left(\mathbf{F}_{q}\right)$ and the conjugacy classes $W(\mathbf{G})^{\sharp}$.

Proof. This is [Ca1, Prop. 3.3.3] though stated a little differently. First, fix a split maximal torus $\mathbf{T}_{0}$ of $\mathbf{G}$. The action of $F$ on $W\left(\mathbf{G}, \mathbf{T}_{0}\right)$ is trivial since $\mathbf{T}_{0}$ is split, so $F$-conjugacy classes of $W\left(\mathbf{G}, \mathbf{T}_{0}\right)$ in [Ca1] are the same as usual conjugacy classes. The equivalence of our statement and Carter's then follows using $W(\mathbf{G}, \mathbf{T})^{\sharp}=W\left(\mathbf{G}, \mathbf{T}_{0}\right)^{\sharp}$ and Proposition 2.1(iii).

We also recall that for any connected reductive group $\mathbf{G} / \mathbf{F}_{q}$, we have

$$
(q-1)^{\operatorname{dim} \mathbf{G}} \leqslant\left|\mathbf{G}\left(\mathbf{F}_{q}\right)\right| \leqslant(q+1)^{\operatorname{dim} \mathbf{G}},
$$

as follows from the formula of Steinberg for $\left|\mathbf{G}\left(\mathbf{F}_{q}\right)\right|$ (see, e.g., [Ca1, p. 75, Prop. 3.3.5]).

The next lemma is well-known, and essentially follows from Lang-Weil estimates in our application, but since we think of this as a fact about finite groups of Lie type, and not about reductions of groups over numbers fields, we give the details (the argument is, in any case, more elementary than the use of the Lang-Weil bounds).

Lemma 4.5. With notation as above, we have

$$
\left|\mathbf{G}\left(\mathbf{F}_{q}\right)_{s r}\right|=\left|\mathbf{G}\left(\mathbf{F}_{q}\right)\right|\left(1+O\left(q^{-1}\right)\right),
$$

where the implicit constant depends only on the type of $\mathbf{G}$.

Proof. As already observed, any element in $\mathbf{G}\left(\mathbf{F}_{q}\right)_{s r}$ lies in a unique maximal torus of $\mathbf{G}$. Hence we have

$$
\left|\mathbf{G}\left(\mathbf{F}_{q}\right)_{s r}\right|=\sum_{\mathbf{T} \in \mathcal{T}}\left|\mathbf{T}\left(\mathbf{F}_{q}\right) \cap \mathbf{G}\left(\mathbf{F}_{q}\right)_{s r}\right|
$$

where $\mathcal{T}$ is the set of ( $F$-stable) maximal tori in $\mathbf{G}$. 
Now fix a maximal torus $\mathbf{T} \in \mathcal{T}$, and let $\Phi=\Phi(\mathbf{G}, \mathbf{T})$ be the set of roots of $\mathbf{G}$ with respect to T. Fix an element $x \in \mathbf{T}\left(\mathbf{F}_{q}\right)$ that is not regular in $\mathbf{G}$. Then there exists a root $\alpha \in \Phi$ such that $\alpha(x)=1$ [Bo, III.12.2]. So let $A$ be the (non-empty) set of roots $\alpha \in \Phi$ for which $\alpha(x)=1$, and define the algebraic subgroup

$$
\mathbf{D}_{A}=\bigcap_{\alpha \in A} \operatorname{ker} \alpha
$$

of $\mathbf{T}$. Since $A$ is $F$-stable, the group $\mathbf{D}_{A}$ is defined over $\mathbf{F}_{q}$ and $x \in \mathbf{D}_{A}\left(\mathbf{F}_{q}\right)$. We thus have

$$
\mid\left\{x \in \mathbf{T}\left(\mathbf{F}_{q}\right): x \text { is not regular in } \mathbf{G}\right\}\left|\leqslant \sum_{A}\right| \mathbf{D}_{A}\left(\mathbf{F}_{q}\right) \mid,
$$

where the sum is over all non-empty $F$-stable subsets $A \subseteq \Phi$. For any such subset $A \subseteq \Phi$, we claim that $\left|\mathbf{D}_{A}\left(\mathbf{F}_{q}\right)\right|=O\left(q^{r-1}\right)$ where $r$ is the dimension of $\mathbf{T}$ and the implied constant depends only on the type of $\mathbf{G}$. Assuming this for now, we have

$$
\mid\left\{x \in \mathbf{T}\left(\mathbf{F}_{q}\right): x \text { is not regular in } \mathbf{G}\right\} \mid \ll \sum_{A} q^{r-1} \ll q^{r-1}
$$

where the implied constant again depends only on the type of $\mathbf{G}$, and hence $\left|\mathbf{T}\left(\mathbf{F}_{q}\right) \cap \mathbf{G}\left(\mathbf{F}_{q}\right)_{s r}\right|=$ $\left|\mathbf{T}\left(\mathbf{F}_{q}\right)\right|+O\left(q^{r-1}\right)$. Applying (4.1) to $\mathbf{T}$, we get

$$
\left|\mathbf{T}\left(\mathbf{F}_{q}\right) \cap \mathbf{G}\left(\mathbf{F}_{q}\right)_{s r}\right|=q^{r}+O\left(q^{r-1}\right) .
$$

We now return to (4.2). According to a theorem of Steinberg [Ca1, Th. 3.4.1], we have $|\mathcal{T}|=q^{2 N}$ where $N$ is the number of positive roots of $\mathbf{G}$, so

$$
\left|\mathbf{G}\left(\mathbf{F}_{q}\right)_{s r}\right|=\sum_{\mathbf{T} \in \mathcal{T}}\left|\mathbf{T}\left(\mathbf{F}_{q}\right) \cap \mathbf{G}\left(\mathbf{F}_{q}\right)_{s r}\right|=|\mathcal{T}|\left(q^{r}+O\left(q^{r-1}\right)\right)=q^{2 N+r}+O\left(q^{2 N+r-1}\right)
$$

where the implied constant depends only on the type of $\mathbf{G}$. The desired estimate for $\left|\mathbf{G}\left(\mathbf{F}_{q}\right)_{s r}\right|$ follows by noting that $2 N+r=\operatorname{dim} \mathbf{G}$ and applying (4.1) to $\mathbf{G}$.

It remains to show that for a fixed maximal torus $\mathbf{T}$ and a non-empty $F$-stable set $A$ of roots of $\mathbf{G}$ relative to $\mathbf{T}$, we have $\left|\mathbf{D}_{A}\left(\mathbf{F}_{q}\right)\right|=O\left(q^{r-1}\right)$ where the implied constant depends only on the type of $\mathbf{G}$. Since the connected component of the identity of a diagonalizable group is a torus such that

$$
\left|\mathbf{D}_{A}^{0}\left(\mathbf{F}_{q}\right)\right| \leqslant(q+1)^{\operatorname{dim} \mathbf{D}_{A}} \leqslant(q+1)^{r-1}
$$

it is enough to show that the number of (geometric) connected components of $\mathbf{D}_{A}$ is bounded in terms of the type of $\mathbf{G}$ only (note that $\operatorname{dim} \mathbf{D}_{A}<\operatorname{dim} \mathbf{T}$, since $A$ is non-empty). From the exact sequence

$$
1 \rightarrow \operatorname{ker}(\alpha) \rightarrow \mathbf{T} \stackrel{\alpha}{\longrightarrow} \mathbf{G}_{m} \rightarrow 1
$$

for $\alpha \in \Phi$, and the dual exact sequence

$$
0 \rightarrow \mathbf{Z} \rightarrow X(\mathbf{T}) \rightarrow X(\operatorname{ker}(\alpha)) \rightarrow 0
$$

of abelian groups of finite rank (see [Bo, III.8.12]), we find that the character group of $X\left(\mathbf{D}_{A}\right)$ is

$$
X\left(\mathbf{D}_{A}\right) \simeq X(\mathbf{T}) /\langle\mathbf{Z} \alpha \mid \alpha \in A\rangle .
$$

The fundamental structure theory of reductive groups shows that the subgroup $\Psi$ of $X(\mathbf{T})$ generated by the roots $\Phi$ together with a basis of the characters of the center of $\mathbf{G}$ is of bounded index in $X(\mathbf{T})$, the bound depending only on the type of $\mathbf{G}$ (see, e.g., [Ca1, 1.11]). Thus the size of the torsion subgroup of $X\left(\mathbf{D}_{A}\right)$ differs from that of

$$
\Psi_{A}=\Psi /\langle\mathbf{Z} \alpha \mid \alpha \in A\rangle,
$$

only by a bound depending only on the type of $\mathbf{G}$. Moreover, $\Psi_{A}$ is defined purely in terms of the root datum, and therefore only depends on the type of $\mathbf{G}$. Thus, the result follows. 
Proof of Proposition 4.1. Fix a conjugacy class $C \in W(\mathbf{G})^{\sharp}$ and let $\mathscr{T}_{C}$ be the set of maximal tori $\mathbf{T}$ of $\mathbf{G}$ for which $\theta(\mathbf{T})=C$. Since a regular semisimple element of $\mathbf{G}$ lies in a unique maximal torus, we have

$$
\begin{aligned}
\frac{\left|\left\{g \in \mathbf{G}\left(\mathbf{F}_{q}\right)_{s r}: \theta(g)=C\right\}\right|}{\left|\mathbf{G}\left(\mathbf{F}_{q}\right)\right|} & =\sum_{\mathbf{T} \in \mathscr{T}_{C}} \frac{\left|\mathbf{T}\left(\mathbf{F}_{q}\right) \cap \mathbf{G}\left(\mathbf{F}_{q}\right)_{s r}\right|}{\left|\mathbf{G}\left(\mathbf{F}_{q}\right)\right|} \\
& =\sum_{\mathbf{T} \in \mathscr{T}_{C}} \frac{\left|\mathbf{T}\left(\mathbf{F}_{q}\right)\right|}{\left|\mathbf{G}\left(\mathbf{F}_{q}\right)\right|}+O\left(\frac{\left|\mathbf{G}\left(\mathbf{F}_{q}\right)-\mathbf{G}\left(\mathbf{F}_{q}\right)_{s r}\right|}{\left|\mathbf{G}\left(\mathbf{F}_{q}\right)\right|}\right) \\
& =\sum_{\mathbf{T} \in \mathscr{T}_{C}} \frac{\left|\mathbf{T}\left(\mathbf{F}_{q}\right)\right|}{\left|\mathbf{G}\left(\mathbf{F}_{q}\right)\right|}+O\left(q^{-1}\right)
\end{aligned}
$$

where the last line uses Lemma 4.5 and the implicit constant depends only on the type of $\mathbf{G}$. It thus suffices to show that

$$
\frac{1}{\left|\mathbf{G}\left(\mathbf{F}_{q}\right)\right|} \sum_{\mathbf{T} \in \mathscr{T}_{C}}\left|\mathbf{T}\left(\mathbf{F}_{q}\right)\right|=\frac{|C|}{|W(\mathbf{G})|} .
$$

By Lemma 4.4, any two tori in $\mathscr{T}_{C}$ are $\mathbf{G}\left(\mathbf{F}_{q}\right)$-conjugate. So after fixing a $\mathbf{T}_{1} \in \mathscr{T}_{C}$ (that $\mathscr{T}_{C} \neq \emptyset$ is part of Lemma 4.4), we have

$$
\left|\mathscr{T}_{C}\right|=\frac{\left|\mathbf{G}\left(\mathbf{F}_{q}\right)\right|}{\left|N_{\mathbf{G}}\left(\mathbf{T}_{1}\right)\left(\mathbf{F}_{q}\right)\right|}
$$

(the denominator being the order of the stabilizer of $\mathbf{T}_{1}$ under $\mathbf{G}\left(\mathbf{F}_{q}\right)$-conjugation) and hence

$$
\frac{1}{\left|\mathbf{G}\left(\mathbf{F}_{q}\right)\right|} \sum_{\mathbf{T} \in \mathscr{T}_{C}}\left|\mathbf{T}\left(\mathbf{F}_{q}\right)\right|=\frac{1}{\left|\mathbf{G}\left(\mathbf{F}_{q}\right)\right|}\left|\mathscr{T}_{C}\right|\left|\mathbf{T}_{1}\left(\mathbf{F}_{q}\right)\right|=\frac{\left|\mathbf{T}_{1}\left(\mathbf{F}_{q}\right)\right|}{\left|N_{\mathbf{G}}\left(\mathbf{T}_{1}\right)\left(\mathbf{F}_{q}\right)\right|}
$$

By Proposition 3.3.6 of [Ca1], we have $\left|N_{\mathbf{G}}\left(\mathbf{T}_{1}\right)\left(\mathbf{F}_{q}\right) / \mathbf{T}_{1}\left(\mathbf{F}_{q}\right)\right|=\left|C_{W\left(\mathbf{G}, \mathbf{T}_{0}\right)}(w)\right|$ where $\mathbf{T}_{0}$ is a split maximal torus of $\mathbf{G}, w \in W\left(\mathbf{G}, \mathbf{T}_{0}\right)$ lies in $C \in W\left(\mathbf{G}, \mathbf{T}_{0}\right)^{\sharp}=W(\mathbf{G})^{\sharp}$, and $C_{W\left(\mathbf{G}, \mathbf{T}_{0}\right)}(w)$ is the centralizer of $w$ in $W\left(\mathbf{G}, \mathbf{T}_{0}\right)$ (the action of $F$ on $W\left(\mathbf{G}, \mathbf{T}_{0}\right)$ is trivial since $\mathbf{T}_{0}$ is split, so the $F$-centralizers in [Ca1] are the same as standard centralizers). Since $|W(\mathbf{G})|=|C| \cdot\left|C_{W\left(\mathbf{G}, \mathbf{T}_{0}\right)}(w)\right|$, the desired formula follows.

It will be important for our application to have uniform bounds and have estimates for those elements lying in certain special cosets in $\mathbf{G}\left(\mathbf{F}_{q}\right)$. Let $\varphi: \mathbf{G}^{s c} \rightarrow \mathbf{G}$ be the universal cover of $\mathbf{G}$ (as an algebraic group), the group and morphism are also defined over $\mathbf{F}_{q}$. The semisimple group $\mathbf{G}^{s c}$ is simply connected and the kernel $\pi_{1}$ of $\varphi$ is a finite group scheme contained in the center of $\mathbf{G}^{s c}$. Our refined equidistribution result is the following.

Proposition 4.6. Let $\mathbf{G}$ be a split semisimple group over $\mathbf{F}_{q}$. Let $\kappa$ be a coset of $\varphi\left(\mathbf{G}^{s c}\left(\mathbf{F}_{q}\right)\right)$ in $\mathbf{G}\left(\mathbf{F}_{q}\right)$. Then for each $C \in W(\mathbf{G})^{\sharp}$, we have

$$
\frac{\left|\left\{g \in \kappa \cap \mathbf{G}\left(\mathbf{F}_{q}\right)_{s r}: \theta(g)=C\right\}\right|}{|\kappa|}=\frac{|C|}{|W(\mathbf{G})|}\left(1+O\left(q^{-1}\right)\right)
$$

where the implicit constant depends only on the type of $\mathbf{G}$.

We start with another simple lemma.

Lemma 4.7. Let $\kappa$ be a coset of $\varphi\left(\mathbf{G}^{s c}\left(\mathbf{F}_{q}\right)\right)$ in $\mathbf{G}\left(\mathbf{F}_{q}\right)$. Then for any maximal torus $\mathbf{T}$ of $\mathbf{G}$, we have

$$
\frac{\left|\mathbf{T}\left(\mathbf{F}_{q}\right) \cap \kappa\right|}{|\kappa|}=\frac{\left|\mathbf{T}\left(\mathbf{F}_{q}\right)\right|}{\left|\mathbf{G}\left(\mathbf{F}_{q}\right)\right|}
$$


Proof. The short exact sequence $1 \rightarrow \pi_{1} \rightarrow \mathbf{G}^{s c} \stackrel{\varphi}{\rightarrow} \mathbf{G} \rightarrow 1$ gives the following long exact sequence in Galois cohomology,

$$
1 \rightarrow \pi_{1}\left(\mathbf{F}_{q}\right) \rightarrow \mathbf{G}^{s c}\left(\mathbf{F}_{q}\right) \stackrel{\varphi}{\rightarrow} \mathbf{G}\left(\mathbf{F}_{q}\right) \stackrel{\delta}{\rightarrow} H^{1}\left(\mathbf{F}_{q}, \pi_{1}\right) \rightarrow 1,
$$

since $H^{1}\left(\mathbf{F}_{q}, \mathbf{G}^{s c}\right)=1$ by Steinberg's theorem [St1, 1.9]. Thus there exists an element $\kappa_{0} \in$ $H^{1}\left(\mathbf{F}_{q}, \pi_{1}\right)$ such that $g \in \mathbf{G}\left(\mathbf{F}_{q}\right)$ lies in $\kappa$ if and only if $\delta(g)=\kappa_{0}$. Since $\pi_{1}$ is contained in the center of $\mathbf{G}^{s c}$, there is a maximal torus $\mathbf{T}^{s c}$ of $\mathbf{G}^{s c}$ giving an exact sequence $1 \rightarrow \pi_{1} \rightarrow \mathbf{T}^{s c} \stackrel{\varphi}{\rightarrow} \mathbf{T} \rightarrow 1$ and a long exact sequence

$$
1 \rightarrow \pi_{1}\left(\mathbf{F}_{q}\right) \rightarrow \mathbf{T}^{s c}\left(\mathbf{F}_{q}\right) \stackrel{\varphi}{\rightarrow} \mathbf{T}\left(\mathbf{F}_{q}\right) \stackrel{\delta^{\prime}}{\rightarrow} H^{1}\left(\mathbf{F}_{q}, \pi_{1}\right) \rightarrow 1 .
$$

The homomorphism $\delta^{\prime}$ agrees with the homomorphism $\delta$ when restricted to $\mathbf{T}\left(\mathbf{F}_{q}\right)$. Therefore,

$$
\frac{\left|\mathbf{T}\left(\mathbf{F}_{q}\right) \cap \kappa\right|}{\left|\mathbf{T}\left(\mathbf{F}_{q}\right)\right|}=\frac{\left|\left\{t \in \mathbf{T}\left(\mathbf{F}_{q}\right): \delta^{\prime}(t)=\kappa_{0}\right\}\right|}{\left|\mathbf{T}\left(\mathbf{F}_{q}\right)\right|}=\frac{1}{\left|H^{1}\left(\mathbf{F}_{q}, \pi_{1}\right)\right|}
$$

and

$$
\frac{|\kappa|}{\left|\mathbf{G}\left(\mathbf{F}_{q}\right)\right|}=\frac{\left|\left\{g \in \mathbf{G}\left(\mathbf{F}_{q}\right): \delta(t)=\kappa_{0}\right\}\right|}{\left|\mathbf{G}\left(\mathbf{F}_{q}\right)\right|}=\frac{1}{\left|H^{1}\left(\mathbf{F}_{q}, \pi_{1}\right)\right|}
$$

Proof of Proposition 4.6. Fix a conjugacy class $C \in W(\mathbf{G})^{\sharp}$ and let $\mathscr{T}_{C}$ be the set of maximal tori $\mathbf{T}$ of $\mathbf{G}$ for which $\theta(\mathbf{T})=C$. Since a regular semisimple element of $\mathbf{G}$ lies in a unique maximal torus, we have

$$
\begin{aligned}
\frac{\left|\left\{g \in \kappa \cap \mathbf{G}\left(\mathbf{F}_{q}\right)_{s r}: \theta(g)=C\right\}\right|}{|\kappa|} & =\sum_{\mathbf{T} \in \mathscr{T}_{C}} \frac{\left|\kappa \cap \mathbf{T}\left(\mathbf{F}_{q}\right) \cap \mathbf{G}\left(\mathbf{F}_{q}\right)_{s r}\right|}{|\kappa|} \\
& =\sum_{\mathbf{T} \in \mathscr{T}_{C}} \frac{\left|\kappa \cap \mathbf{T}\left(\mathbf{F}_{q}\right)\right|}{|\kappa|}+O\left(\frac{\left|\mathbf{G}\left(\mathbf{F}_{q}\right)-\mathbf{G}\left(\mathbf{F}_{q}\right)_{s r}\right|}{|\kappa|}\right) \\
& =\sum_{\mathbf{T} \in \mathscr{T}_{C}} \frac{\left|\kappa \cap \mathbf{T}\left(\mathbf{F}_{q}\right)\right|}{|\kappa|}+O\left(q^{-1}\right)
\end{aligned}
$$

where the last line uses Lemma 4.5 and $\left|\mathbf{G}\left(\mathbf{F}_{q}\right)\right| /|\kappa|=O(1)$ (the implicit constants depend only on the type of $\mathbf{G})$. By Lemma 4.7, we have

$$
\frac{\left|\left\{g \in \kappa \cap \mathbf{G}\left(\mathbf{F}_{q}\right)_{s r}: \theta(g)=C\right\}\right|}{|\kappa|}=\left|\mathbf{G}\left(\mathbf{F}_{q}\right)\right|^{-1} \sum_{\mathbf{T} \in \mathscr{T}_{C}}\left|\mathbf{T}\left(\mathbf{F}_{q}\right)\right|+O\left(q^{-1}\right) .
$$

This completes the proof since we have already proved that

$$
\left|\mathbf{G}\left(\mathbf{F}_{q}\right)\right|^{-1} \sum_{\mathbf{T} \in \mathscr{T}_{C}}\left|\mathbf{T}\left(\mathbf{F}_{q}\right)\right|=|C| /|W(\mathbf{G})|
$$

in the course of the proof of Proposition 4.6.

\section{Sieve for Random Walks on SEmisimple Algebraic groups}

To prove our main results in the next section, we will use sieve methods. We first consider in this section the problem of obtaining a general (upper-bound) sieve result for "random" elements of an arithmetic group in a semisimple group over a number field.

To give a meaning to "random" elements in $\mathbf{G}$, we use random walks, as in [K, Ch. 7] (but see Section 7 for comments on other possibilities). This involves a fair amount of notation, but is otherwise quite convenient.

In all this section, we therefore consider to have fixed the following data: 
- A number field $k$;

- A connected semisimple algebraic group $\mathbf{G} / k$ (not necessarily split);

- An arithmetic subgroup $\Gamma \subset \mathbf{G}(k)$ of $\mathbf{G}$, as defined in the introduction, e.g.,

$$
\Gamma=\rho(\mathbf{G}(k)) \cap \mathrm{GL}\left(N, \mathbf{Z}_{k}\right)
$$

for some faithful representation $\rho: \mathbf{G} \hookrightarrow \operatorname{GL}(N)$ over $k$.

- A finite symmetric (i.e., $s \in S$ implies $s^{-1} \in S$ ) generating $S$ set of $\Gamma$ (the group $\Gamma$ is finitely generated $^{1}$ by a theorem of Borel, see e.g. [PR, Th. 4.17 (2)]); we will always assume that the pair $(\Gamma, S)$ is balanced, by which we mean that either (i) $1 \in S$, or (ii) there exists no non-trivial homomorphism $\Gamma \rightarrow \mathbf{Z} / 2 \mathbf{Z}$ (this is in order to avoid possible issues with bipartite Cayley graphs, see $[K, \S 7.4]$ for a discussion of this point; we thank the referee for having reminded us of this issue);

- A sequence $\left(\xi_{n}\right)$ of independent, identically distributed, random variables, defined on some probability space $(\Omega, \Sigma, \mathbf{P})$, taking values in $S$ :

$$
\xi_{n}: \Omega \rightarrow S,
$$

such that $p(s)=\mathbf{P}\left(\xi_{n}=s\right)>0$ for all $s \in S$, and $p(s)=p\left(s^{-1}\right)$ for all $s$.

Example 5.1. Readers not familiar with the general theory may take:

- The field $k=\mathbf{Q}$;

- The group $\mathbf{G}=\mathrm{SL}(N), N \geqslant 2$, with $\rho$ the inclusion in $\mathrm{GL}(N)$;

- The arithmetic group $\Gamma=\operatorname{SL}(N, \mathbf{Z})$;

- The system of generators $S$ of elementary matrices $\operatorname{Id} \pm E_{i, j}$ for distinct $i, j \in\{1, \ldots, n\}$ where $E_{i, j}$ has zero in all entries except for the $(i, j)$-th where it is one; for $N=2$, we add 1 to $S$ in order for $(\Gamma, S)$ to be balanced (when $N \geqslant 3$, there is no non-trivial map $\mathrm{SL}(N, \mathbf{Z}) \rightarrow \mathbf{Z} / 2 \mathbf{Z})$

- The probability space $\Omega=\left\{\left(s_{n}\right)_{n \geqslant 1} \mid s_{n} \in S\right\}$, with the product uniform normalized counting measure, $\xi_{n}(\omega)=s_{n}$ for $\omega=\left(s_{n}\right)_{n \geqslant 1} \in \Omega$, so that $p(s)=1 /|S|$ for all $s$.

This is a setting already considered in $[K, \S 7]$. Note however that in that case $\mathbf{G}$ is simply connected, so much of the work needed below to deal with the general case is unnecessary. For a non-simply connected example, one may take $\mathbf{G}=\mathrm{SO}(N, N)$ for $N \geqslant 2$, and $\Gamma=\mathrm{SO}(N, N)(\mathbf{Z})$.

To have a meaningful asymptotic problem, the discrete group $\Gamma$ must be "big enough". It seems that the right way to quantify this in our setting is simply to assume that $\Gamma$ is Zariski-dense in G. By the Borel Density Theorem (see, e.g., [PR, Th. 4.10]), this assumption on the arithmetic group $\Gamma$ can be formulated purely in terms of the semisimple group $\mathbf{G}$ : it means that for any simple component of $\mathbf{G}$, say $\mathbf{H}$, and any real or complex completion $K$ of $k$, the group $\mathbf{H}(K)$ is noncompact for the real or complex topology. In particular, this holds whenever $\mathbf{G}$ is split.

We then define

$$
X_{0}=1 \in \Gamma, \quad X_{n+1}=X_{n} \xi_{n+1},
$$

so that the sequence $\left(X_{n}\right)$ is a random walk on $\Gamma$.

To perform the sieve, we require independence properties of reductions modulo primes of arithmetic groups. This independence is only valid for simply connected groups, and to reduce to this case we use ideas already found in [J] with some new tools.

Let $\varphi: \mathbf{G}^{s c} \rightarrow \mathbf{G}$ be the simply connected covering of $\mathbf{G}$ (as an algebraic group). Both $\mathbf{G}^{s c}$ and $\varphi$ are defined over $k$, so we can define

$$
\Gamma^{s c}=\varphi\left(\varphi^{-1}(\Gamma) \cap \mathbf{G}^{s c}(k)\right) \subset \Gamma .
$$

\footnotetext{
${ }^{1}$ In fact, finitely presented, which is a quite deeper property which we do not need.
} 
It follows from basic facts about arithmetic groups (see, e.g., [PR, Theorem 4.1]) that $\Gamma^{s c}$ is an arithmetic subgroup of $\mathbf{G}$. In fact, since $\Gamma^{s c} \subset \Gamma$, it follows that $\Gamma^{s c}$ has finite index in $\Gamma$.

As recalled in Section 3, there exists a finite set $R_{0}$ of prime ideals of $\mathbf{Z}_{k}$ such that $\mathbf{G}$ has a model defined over the ring $\mathbf{Z}_{k}\left[1 / R_{0}\right]$, and such that any two such models are isomorphic after possibly inverting finitely many more primes. By abuse of notation, we will also denote the fixed model by G. After possibly increasing $R_{0}$, we may assume that $\Gamma \subseteq \mathbf{G}\left(\mathbf{Z}_{k}\left[1 / R_{0}\right]\right)$. In particular, for $\mathfrak{p} \notin R_{0}$, we obtain a well-defined reduction map

$$
\pi_{\mathfrak{p}}: \Gamma \rightarrow \mathbf{G}\left(\mathbf{F}_{\mathfrak{p}}\right)
$$

and similarly for the simply connected cover $\mathbf{G}^{s c}$, and we have homomorphisms

$$
\varphi_{\mathfrak{p}}: \mathbf{G}^{s c}\left(\mathbf{F}_{\mathfrak{p}}\right) \rightarrow \mathbf{G}\left(\mathbf{F}_{\mathfrak{p}}\right) .
$$

The following deep result, called the "Strong Approximation Property", explains why we need to use $\Gamma^{s c}$ : the statement is false, in general, if $\Gamma^{s c}$ is replaced with $\Gamma$ itself.

Proposition 5.2. Let $(k, \mathbf{G}, \Gamma)$ be as given, in particular such that $\Gamma$ is Zariski-dense in $\mathbf{G}$, and let $\mathbf{G}^{s c}, \Gamma^{s c}$ be as defined above. Let

$$
\Gamma_{\mathfrak{p}}^{s c}=\pi_{\mathfrak{p}}\left(\Gamma^{s c}\right),
$$

where $\pi_{\mathfrak{p}}$ is the reduction map defined above for almost all prime ideals of $\mathbf{Z}_{k}$.

Then there exists a finite subset $R \supset R_{0}$ of prime ideals, depending only on $\left(k, \mathbf{G}, \Gamma, R_{0}\right)$, such that for any $\mathfrak{p} \notin R$, we have

$$
\Gamma_{\mathfrak{p}}^{s c}=\varphi_{\mathfrak{p}}\left(\mathbf{G}^{s c}\left(\mathbf{F}_{\mathfrak{p}}\right)\right)
$$

the image of the group of $\mathbf{F}_{\mathfrak{p}}$-rational points of the simply connected covering of $\mathbf{G}$, and moreover the product maps

$$
\Gamma^{s c} \stackrel{\pi_{\mathfrak{p}} \times \pi_{\mathfrak{p}^{\prime}}}{\longrightarrow} \Gamma_{\mathfrak{p}}^{s c} \times \Gamma_{\mathfrak{p}^{\prime}}^{s c}
$$

for $\mathfrak{p} \neq \mathfrak{p}^{\prime}$, both not in $R$, are surjective.

Proof. Results of this type, in varying generality, have been proved by many people, using a wide variety of techniques; see, e.g., the papers of Nori [No, Th. 5.1], Matthews, Vaserstein and Weisfeiler [MVW, Th., p. 515], Weisfeiler [W, §9], Hrushovski and Pillay [HP, Prop. 7.3] (see also the comments in $[\mathrm{PR}, \S 7.5])$. Precisely, we first apply [W, Th. 9.1.1] with data

$$
(k, G, \Gamma)=\left(k, \mathbf{G}^{s c}, \varphi^{-1}(\Gamma)\right)
$$

to deduce that $\varphi^{-1}(\Gamma)$ surjects to $\mathbf{G}^{s c}\left(\mathbf{Z}_{k} / I\right)$ for all integral ideals $I \neq 0$ coprime with some finite set of primes in $\mathbf{Z}_{k}$. Taking $I=\mathfrak{p}$ and composing with $\varphi$ and $\varphi_{\mathfrak{p}}$, respectively, we derive $\Gamma_{\mathfrak{p}}^{s c}=\pi_{\mathfrak{p}}\left(\Gamma^{s c}\right)$ for $\mathfrak{p} \notin R$.

Then, since

$$
\mathbf{G}^{s c}\left(\mathbf{Z}_{k} / \mathfrak{p p}^{\prime}\right)=\mathbf{G}^{s c}\left(\mathbf{Z}_{k} / \mathfrak{p}\right) \times \mathbf{G}^{s c}\left(\mathbf{Z}_{k} / \mathfrak{p}^{\prime}\right)
$$

if $\mathfrak{p} \neq \mathfrak{p}^{\prime}$ are both prime ideals not in $R$ (by a straightforward Chinese Remainder Theorem), it also follows that $\varphi^{-1}(\Gamma)$ surjects onto $\mathbf{G}^{s c}\left(\mathbf{F}_{\mathfrak{p}}\right) \times \mathbf{G}^{s c}\left(\mathbf{F}_{\mathfrak{p}^{\prime}}\right)$ for $\mathfrak{p}$ and $\mathfrak{p}^{\prime}$ both outside $R$, and the final conclusion is obtained by applying again the map $\varphi$.

Remark 5.3. Here is an illustration of failure of this result when the group is not simply connected: let $Q$ be a nondegenerate indefinite quadratic form over $\mathbf{Z}$ and $\mathbf{G}=\mathrm{SO}(Q)$, which is defined over Z. It is a standard fact that the spinor norm of an element in the group of integral points $\operatorname{SO}(Q, \mathbf{Z})$ is \pm 1 (modulo the non zero squares of $\mathbf{Q}^{\times}$). Thus for any prime $p$ congruent to 1 modulo 4 (i.e. for a subset of primes of density $1 / 2)$, the image of $\operatorname{SO}(Q, \mathbf{Z})$ by reduction modulo $p$ equals the spinorial kernel $\Omega\left(n, \mathbf{F}_{p}\right)$ which means that for any $p$ congruent to 1 modulo 4 , the morphism of reduction modulo $p$ fails to be surjective onto $\mathrm{SO}\left(Q, \mathbf{F}_{p}\right)$ and its image has index 2 . 
Since $\Gamma^{s c}$ is of finite index in $\Gamma$, the idea is now to use random walks on the cosets of $\Gamma^{s c}$ in $\Gamma$ in order to perform the sieve. Of course, the original walk we wish to consider is not of this type. One could, as in [J, Section 1.1] decide that it is good enough to deal with each fixed coset separately (using random variables of the type $Y_{n}=\gamma X_{n}$ for a fixed $\gamma$ and a random walk $\left(X_{n}\right)$ on $\Gamma^{s c}$ ), provided we obtain the "same" result, independently of $\gamma$. However, we want to do better. For this, the idea is also suggested by the (easy) case of [K, Prop. 7.11], where random walks on $\operatorname{Sp}(4, \mathbf{Z})$ were studied by reducing to auxiliary random walks (namely $Y_{n}=X_{2 n}$ and $Z_{n}=X_{2 n+1}$ ) on the two cosets in $\operatorname{Sp}(4, \mathbf{Z}) /[\operatorname{Sp}(4, \mathbf{Z}), \operatorname{Sp}(4, \mathbf{Z})]$, when the original walk had the property that every other step was in the non-identity coset.

We do something similar; we don't know exactly when the finitely many cosets $\gamma \in \Gamma^{s c} \backslash \Gamma$ are reached, but we can use probabilistic results to show that every coset is covered essentially equally often. Note that readers not familiar with the basic properties of Markov chains (with countable state space) may wish to assume that $\Gamma^{s c}=\Gamma$ and skip directly to Corollary 5.7 , reading the latter with this assumption in mind. ${ }^{2}$

Let $\mathcal{C}=\Gamma^{s c} \backslash \Gamma$ be the finite set of cosets; we write $g \equiv \gamma$ to state that $g \in \Gamma$ is in the coset $\gamma \in \mathcal{C}$ (instead of $g \in \gamma$ ). Fix representatives $\tilde{\gamma}$ in $\Gamma$ of all $\gamma \in \mathcal{C}$.

Let now $\left(\gamma_{n}\right)$ be the random walk on the finite set $\mathcal{C}=\Gamma^{s c} \backslash \Gamma$ induced from the walk $\left(X_{n}\right)$ on $\Gamma$. In probabilistic terms, $\left(\gamma_{n}\right)$ is a finite Markov chain with Markov kernel

$$
K\left(\gamma, \gamma^{\prime}\right)=\sum_{s \in S, \gamma s=\gamma^{\prime}} p(s)
$$

This Markov chain is irreducible, because the possible steps $S$ of $\left(X_{n}\right)$ have positive probability and generate $\Gamma$, and reversible because the probabilities $p(s)$ satisfy $p(s)=p\left(s^{-1}\right.$ ). The (unique) stationary distribution associated with $\left(\gamma_{n}\right)$ is the uniform distribution on $\mathcal{C}$, i.e., we have

$$
\frac{1}{|\mathcal{C}|} \sum_{\gamma \in \mathcal{C}} K\left(\gamma, \gamma^{\prime}\right)=\frac{1}{|\mathcal{C}|}
$$

for any $\gamma^{\prime} \in \mathcal{C}$. (For basic facts and terminology, we refer to [Sa, §2] or [BW, Ch. II].)

For any $\gamma \in \mathcal{C}$, we define recursively the following sequence of random times

$$
t_{\gamma, j}: \Omega \rightarrow\{0,1,2 \ldots,\} \cup \infty,
$$

which indicate for which successive indices the walk falls in $\gamma$ : first

$$
t_{\gamma, 0}=\min \left\{n \geqslant 0 \mid X_{n} \equiv \gamma\right\},
$$

and for $j \geqslant 0$, we have

$$
t_{\gamma, j+1}= \begin{cases}+\infty & \text { if } t_{\gamma, j}=+\infty \\ \min \left\{n>t_{\gamma, j} \mid X_{n} \equiv \gamma\right\}, & \text { otherwise. }\end{cases}
$$

We then define auxiliary random walks by

$$
Y_{\gamma, j}= \begin{cases}X_{t_{\gamma, j}} \in \gamma & \text { if } t_{\gamma, j}<+\infty \\ \tilde{\gamma} \in \gamma & \text { otherwise }\end{cases}
$$

where (as we will see immediately) the second case is only present for definiteness.

These random walks are then quite similar to the original ones, but (by definition) lie in a single coset of $\Gamma^{s c}$.

\footnotetext{
2 This assumption holds in a number of cases, such as $\mathrm{SL}(m)$ or $\operatorname{Sp}(2 g)$.
} 
Lemma 5.4. With notation as above, we have the following properties:

(1) Almost surely, all the $t_{\gamma, j}$ are finite.

(2) For any $\gamma \in \mathcal{C}$, the sequence $\left(Y_{\gamma, j}\right)_{j \geqslant 0}$ is a random walk on the coset $\gamma$, given by the initial $\gamma$-valued random variable $Y_{\gamma, 0}$, and with steps

$$
\beta_{\gamma, j}=Y_{\gamma, j-1}^{-1} Y_{\gamma, j}
$$

which are $\Gamma^{s c}$-valued, independent and identically distributed; their distribution is given by the rule

$$
\mathbf{P}\left(\beta_{\gamma, j}=g\right)=\sum_{k \geqslant 1} \sum_{\substack{s_{1} \cdots s_{k}=g \\ s_{1} \cdots s_{m} \notin \Gamma^{s c}, m<k}} p\left(s_{1}\right) \cdots p\left(s_{k}\right), \quad \text { for } g \in \Gamma^{s c} .
$$

Moreover, we have $\mathbf{P}\left(\beta_{\gamma, j}=g\right)=\mathbf{P}\left(\beta_{\gamma, j}=g^{-1}\right)$ for any $g \in \Gamma^{s c}$.

Proof. Part (1) is a well-known property of finite irreducible Markov chains (it is possible to go from any coset to the other), see, e.g., [BW, Prop. II.8.1].

Part (2): from (1), we know that the random walk $\left(Y_{\gamma, j}\right)$ is well-defined. Its initial state is $Y_{\gamma, 0}$ by definition. Therefore, it remains to show that the steps

$$
\beta_{\gamma, j}=Y_{\gamma, j-1}^{-1} Y_{\gamma, j}=X_{t_{\gamma, j-1}}^{-1} X_{t_{\gamma, j}}, \quad \text { for } j \geqslant 1
$$

are distributed according to (5.1), are independent, and independent of the initial step $Y_{\gamma, 0}$. This is intuitively natural, and is a fairly standard fact in probability, but we give a certain amount of details for completeness for those readers who have not seen this type of arguments before (see, e.g., [BW, II, Th. 4.1] for similar reasoning).

Of course, $\beta_{\gamma, j}$ is $\Gamma^{s c}$-valued by construction. We will show that the distribution is the one claimed. For $g \in \Gamma^{s c}$, we have

$$
\begin{aligned}
& \mathbf{P}\left(\beta_{\gamma, j}=g\right)=\mathbf{P}\left(X_{t_{\gamma, j}}=X_{t_{\gamma, j-1}} g\right) \\
& =\sum_{k \geqslant 1} \mathbf{P}\left(t_{\gamma, j}=t_{\gamma, j-1}+k, \text { and } X_{t_{\gamma, j-1}+k}=X_{t_{\gamma, j-1}} g\right) \\
& =\sum_{k \geqslant 1} \sum_{\substack{\left(s_{1}, \ldots, s_{k}\right) \in S^{k} \\
g=s_{1} \cdots s_{k}}} \mathbf{P}\left(t_{\gamma, j}=t_{\gamma, j-1}+k \text { and } \xi_{t_{\gamma, j-1}+m}=s_{m}, \text { for } 1 \leqslant m \leqslant k\right) \\
& =\sum_{k \geqslant 1} \sum_{\substack{g=s_{1} \cdots s_{k} \\
s_{1} \cdots s_{m} \notin \Gamma^{s c}, m<k}} \mathbf{P}\left(\xi_{t_{\gamma, j-1}+m}=s_{m}, \text { for } 1 \leqslant m \leqslant k\right)
\end{aligned}
$$

since the condition that $t_{\gamma, j}=t_{\gamma, j-1}+k$ means, by definition of $t_{\gamma, j}$ and the fact that $X_{t_{\gamma, j-1}} \in \Gamma^{s c}$, that none of the intermediate elements

$$
\xi_{t_{\gamma, j-1}+1} \cdots \xi_{t_{\gamma, j-1}+m}=s_{1} \cdots s_{m}
$$

are in $\Gamma^{s c}$ for $1 \leqslant m<k$.

Now we can invoke the strong Markov property of the original random walk [BW, Th. II.4.1], which implies that the random walk defined by

$$
Z_{m}=X_{t_{\gamma, j-1}+m}, \quad \text { for } m \geqslant 0
$$

is itself a random walk on $\Gamma$ with steps which are independent and distributed like the original steps of $\left(X_{n}\right)$. Note that this would be obvious if $t_{\gamma, j-1}$ were constant, ${ }^{3}$ but is false for a general random time: imagine for instance looking at $Z_{m}=X_{T+m}$ where the time $T$ is defined to be the least index $n$ such that $\xi_{n+1}=s$ (for some fixed $s \in S$ ). The suitable property which holds for the

\footnotetext{
${ }^{3}$ In which case it is the Markov property.
} 
random time $t_{\gamma, j-1}$ is that it is a stopping time for the standard filtration associated with $\left(X_{n}\right)$, meaning that the events

$$
\left\{t_{\gamma, j-1}=k\right\}
$$

for any $k \geqslant 0$, are measurable for the $\sigma$-field $\sigma\left(X_{1}, \ldots, X_{k}\right)$ (which is obvious since determining whether $t_{\gamma, j-1}=k$ can be done by looking at the first $k$ steps of the original walk).

¿From this, it follows that

$$
\begin{aligned}
\mathbf{P}\left(\xi_{t_{\gamma, j-1}+m}=s_{m}, \text { for } 1 \leqslant m \leqslant k\right) & =\mathbf{P}\left(\xi_{m}=s_{m}, \text { for } 1 \leqslant m \leqslant k\right) \\
& =p\left(s_{1}\right) \cdots p\left(s_{k}\right),
\end{aligned}
$$

and the distribution property (5.1) then follows from (5.2). The symmetry property of the distribution is obvious.

The independence of the steps $\beta_{\gamma, j}$ is also a consequence of the strong Markov property and computations very similar to the previous one, except for notational complications.

Note that in these auxiliary walks, the initial distribution depends on $\gamma$, not the steps of the walk (though, $\mathcal{C}$ being finite, such a dependency would not affect the remainder of the argument). A further difference with the original walk $\left(X_{n}\right)$ is the feature that the steps $\beta_{\gamma, j}$ are supported on the whole of the discrete group $\Gamma^{s c}$, instead of the original finite set $S$. This is, however, still a symmetric generating set of $\Gamma^{s c}$. It turns out that random walks involving infinite generating sets were also already considered in [J, Introduction and Section 1.2], so we can build on this.

The following general sieve result follows quite simply from the theory developed in $[K, \S 7]$ and the adjustments in $[\mathrm{J}]$.

Proposition 5.5. Let $(k, \mathbf{G}, \Gamma)$ be given as before, and define $\Gamma^{s c}, \mathcal{C}, \Gamma_{\mathfrak{p}}^{s c}, \pi_{\mathfrak{p}}: \Gamma^{s c} \rightarrow \Gamma_{\mathfrak{p}}^{s c}$ as above.

Let $\left(Y_{j}\right), j \geqslant 0$, be a random walk on a fixed coset $\gamma \in \mathcal{C}$ of $\Gamma^{s c}$, with initial step $Y_{0}$ and with independent, identically distributed steps $\left(\beta_{j}\right), j \geqslant 1$, such that the support of the law of the $\beta_{j}$ is a generating set of $\Gamma^{s c}$, and

$$
\mathbf{P}\left(\beta_{j}=g\right)=\mathbf{P}\left(\beta_{j}=g^{-1}\right), \quad \text { for } j \geqslant 1, g \in \Gamma^{s c} .
$$

Assume that $\left(\Gamma^{s c}, S\right)$ is a balanced pair: either $\mathbf{P}\left(\beta_{j}=1\right)>0$, or there is no surjection $\Gamma^{s c} \rightarrow$ $\mathbf{Z} / 2 \mathbf{Z}$.

There exists a finite set $R$ of prime ideals in $\mathbf{Z}_{k}$, depending only on $\Gamma$, and constants $c>0$ and $A \geqslant 0$, depending only on $k, \Gamma$ and the distribution of the steps $\left(\beta_{j}\right)$ such that the following holds: for any choice of subsets

$$
\Omega_{\mathfrak{p}} \subset \tilde{\gamma} \Gamma_{\mathfrak{p}}^{s c},
$$

invariant under $\mathbf{G}\left(\mathbf{F}_{\mathfrak{p}}\right)$-conjugation, with $\Omega_{\mathfrak{p}}=\emptyset$ if $\mathfrak{p} \in R$, we have

$$
\mathbf{P}\left(\pi_{\mathfrak{p}}\left(Y_{j}\right) \notin \Omega_{\mathfrak{p}} \text { for } N \mathfrak{p} \leqslant L\right) \leqslant\left(1+L^{A} e^{-c j}\right) V^{-1}
$$

for any $L \geqslant 2$, where

$$
V=\sum_{N \mathfrak{p} \leqslant L} \frac{\left|\Omega_{\mathfrak{p}}\right|}{\left|\Gamma_{\mathfrak{p}}^{s c}\right|} .
$$

Proof. The main ingredient, beside Proposition 5.2, is the following:

[Property $(\tau)]$ The group $\Gamma^{s c}$ has Property $(\tau)$ (in the sense of Lubotzky) with respect to the family of its congruence subgroups, and in particular with respect to the family of subgroups of the type

$$
\left(\operatorname{ker}\left(\pi_{\mathfrak{p}} \times \pi_{\mathfrak{p}^{\prime}}\right)\right)
$$

where $\mathfrak{p}$ and $\mathfrak{p}^{\prime}$ run over all prime ideals not in $R$. This conjecture of Lubotzky and Zimmer was proved by Clozel [Cl]. 
We then apply the general methods in $[\mathrm{K}, \mathrm{Ch} .3, \mathrm{Ch} .7]$ (compare with [J, Section 1]). More precisely, we first note that, from Property $(\tau)$, there exists a finite subset (say $S^{\prime}$ ) of $\Gamma^{s c}$ with

$$
\min _{s \in S^{\prime}} \mathbf{P}\left(\beta_{j}=s\right)>0
$$

and $\delta>0$ with the following property: for any finite-dimensional unitary representation

$$
\Gamma^{s c} \stackrel{\rho}{\longrightarrow} U(N, \mathbf{C})
$$

that factors through some product of "prime" congruence groups, i.e., $\rho$ is given by

$$
\Gamma^{s c} \rightarrow \Gamma^{s c} / \operatorname{ker}\left(\pi_{\mathfrak{p}} \times \pi_{\mathfrak{p}^{\prime}}\right) \stackrel{\rho^{\prime}}{\longrightarrow} U(N, \mathbf{C}),
$$

we have

$$
\min _{s \in S^{\prime}}\{\|\rho(s) v-v\|\} \geqslant \delta\|v\|, \quad v \in \mathbf{C}^{N}
$$

provided there is no vector $v \neq 0$ which is invariant under $\rho$.

With this, one can follow the proof of [K, Prop. 7.2], or [J, Prop. 5] to obtain the large sieve bound.

If we use this technique to control a random walk on $\Gamma$ itself by splitting into auxiliary walks, this proposition requires one extra piece of information to be useful: namely, the estimate in terms of $j$ must be transformed into information in terms of the parameter $n$ of the original walk. Intuitively, since there are $|\mathcal{C}|$ different cosets, and the random walk on $\mathcal{C}$ mixes very quickly (it is a finite irreducible Markov chain), converging to the stationary uniform distribution on $\mathcal{C}$ (all cosets being equally likely), we expect that $X_{n}$ is, roughly, the $j$-th step of the auxiliary random walk $Y_{\gamma_{n}, j}$ for an index $j$ close to $n /|\mathcal{C}|$. The following result makes this precise:

Lemma 5.6. Let $\left(k, \mathbf{G}, \Gamma, S,\left(X_{n}\right)\right)$ be given as before, and let $\left(\mathbf{G}^{s c}, \mathcal{C},\left(\gamma_{n}\right),\left(t_{\gamma, j}\right)_{\gamma, j},\left(Y_{\gamma, j}\right)\right)$ be defined as above.

For $n \geqslant 0$, let $\iota_{n}$ be the random index such that

$$
X_{n}=Y_{\gamma_{n}, \iota_{n}} .
$$

Then we have

$$
\mathbf{P}\left(\iota_{n}<\frac{1}{2} \frac{n+1}{|\mathcal{C}|}\right) \ll \exp (-c n),
$$

for all $n \geqslant 0$ and some constant $c>0$, depending only on $\mathcal{C}$ and the distribution of the steps of the Markov chain $\gamma_{n}$, as does the implied constant.

Proof. We can express $\iota_{n}$ concisely by

$$
\iota_{n}=\left|\left\{i \mid 0 \leqslant i \leqslant n, X_{i} \equiv X_{n}\right\}\right|, \quad \text { so } \quad \frac{\iota_{n}}{n+1}=\frac{1}{n+1} \sum_{0 \leqslant i \leqslant n} \mathbf{1}_{\left\{\gamma_{n}\right\}}\left(\gamma_{i}\right) .
$$

Now fix $\gamma \in \mathcal{C}$ instead, and consider the deterministic variant

$$
\kappa_{\gamma, n}=\frac{1}{n+1} \sum_{0 \leqslant i \leqslant n} \mathbf{1}_{\gamma}\left(\gamma_{i}\right)
$$

¿From basic properties of Markov chains, we know that

$$
\lim _{n \rightarrow+\infty} \mathbf{P}\left(\gamma_{n}=\gamma\right)=\frac{1}{|\mathcal{C}|}
$$

by equidistribution of the random walk $\left(\gamma_{n}\right)$ on the finite set $\mathcal{C}$ (in particular, this does not depend on $\gamma$ ). Noting that

$$
\mathbf{E}\left(\mathbf{1}_{\gamma}\left(\gamma_{i}\right)\right)=\mathbf{P}\left(\gamma_{i}=\gamma\right)
$$


we may expect by results like the law of large numbers that $\kappa_{\gamma, n}$ is usually "close" to $\frac{1}{|\mathcal{C}|}$, which makes it clear intuitively why the probability we look for should be small.

However, because the random variables $\mathbf{1}_{\gamma}\left(\gamma_{i}\right)$ are not independent, we can not simply apply the standard results about sums of independent random variables. But because the convergence to equidistribution (5.4) is exponentially fast, fairly classical works in probability theory have extended the basic convergence results (weak and strong law of large numbers, central limit theorem, large deviations results) to this context.

We precisely need a large deviation result, which in the simplest (classical) context is the Chernov bound. Here we quote for concreteness from the explicit result in [L], though general bounds go back to Miller, Gillman, Donsker and Varadhan (and Lezaud's result has been improved in some contexts by León and Perron).

From [L, Th. 1.1, Remark 3], we obtain

$$
\mathbf{P}\left(\kappa_{\gamma, n}<\frac{1}{2} \frac{n+1}{|\mathcal{C}|}\right) \leqslant e^{\beta / 5}|\mathcal{C}|^{1 / 2} \exp \left(-\frac{\beta(n+1)}{12 \cdot(2|\mathcal{C}|)^{2}}\right)
$$

where $\beta>0$ is the spectral gap of the Markov chain $\left(\gamma_{n}\right)$ (precisely, apply [L, Remark 3] with the data given by

$$
\left(G, X_{i}, \pi\right)=\left(\mathcal{C}, \gamma_{i+1} \text {, the uniform distribution on } \mathcal{C}\right)
$$

so that $N_{q}$ in loc. cit. is bounded by $\sqrt{|\mathcal{C}|}$, and the function $f$ is given by

$$
f(g)=\frac{1}{|\mathcal{C}|}-\mathbf{1}_{\gamma}(g), \quad \text { for } g \in \mathcal{C},
$$

while the constant denoted $\gamma$ in loc. cit. is $\left.(2|\mathcal{C}|)^{-1}\right)$. Note the upper bound we derived does not depend on $\gamma \in \mathcal{C}$.

Finally, to come back to the actual index $\iota_{n}$, we simply write

$$
\begin{aligned}
\mathbf{P}\left(\iota_{n}<\frac{1}{2} \frac{n+1}{|\mathcal{C}|}\right) & =\sum_{\gamma \in \mathcal{C}} \mathbf{P}\left(\kappa_{\gamma, n}<\frac{1}{2} \frac{n+1}{|\mathcal{C}|} \text { and } \gamma_{n}=\gamma\right) \\
& \leqslant \sum_{\gamma \in \mathcal{C}} \mathbf{P}\left(\kappa_{\gamma, n}<\frac{1}{2} \frac{n+1}{|\mathcal{C}|}\right) \\
& \leqslant e^{\beta / 5}|\mathcal{C}|^{3 / 2} \exp \left(-\frac{\beta(n+1)}{12 \cdot(2|\mathcal{C}|)^{2}}\right) .
\end{aligned}
$$

Cleaning up the constants, this clearly implies the result as stated (and is in fact much more precise).

This lemma means that, except for exceptions occurring with exponentially decaying probability, the sieve statement for the auxiliary walks leads to a sieve statement for the original one, where the dependency on the lenght behaves as expected.

Corollary 5.7. Let $k, \mathbf{Z}_{k}, \mathbf{G}, \Gamma, \Gamma^{s c}, \pi_{\mathfrak{p}}, \Gamma_{\mathfrak{p}}^{s c}, \mathcal{C}$ be as above, in particular $\Gamma$ is Zariski-dense in G.

Let $\left(X_{n}\right), n \geqslant 0$, be a random walk on $\Gamma$ starting at the origin with independent, identically distributed steps $\xi_{n}, n \geqslant 1$, supported on a finite symmetric generating set $S$ of $\Gamma$ with

$$
\mathbf{P}\left(\xi_{n}=s\right)=\mathbf{P}\left(\xi_{n}=s^{-1}\right)>0, \quad \text { for } n \geqslant 1, s \in S,
$$

and such that $(\Gamma, S)$ is balanced in the sense described at the beginning of Section 5.

There exists a finite set $R$ of prime ideals in $\mathbf{Z}_{k}$, depending only on $\Gamma$, and constants $c>0$ and $A \geqslant 0, B \geqslant 0$, depending only on $k, \Gamma$ and the distribution of the steps $\left(\xi_{n}\right)$ such that the following holds: for any choice of subsets

$$
\Omega_{\mathfrak{p}} \subset \Gamma_{\mathfrak{p}}=\pi_{\mathfrak{p}}(\Gamma)
$$


invariant under $\Gamma_{\mathfrak{p}}$-conjugation, with $\Omega_{\mathfrak{p}}=\emptyset$ if $\mathfrak{p} \in R$, we have

$$
\mathbf{P}\left(\pi_{\mathfrak{p}}\left(X_{n}\right) \notin \Omega_{\mathfrak{p}} \text { for } N \mathfrak{p} \leqslant L\right) \leqslant B e^{-c n}+n\left(1+L^{A} e^{-c n}\right) \sum_{\gamma \in \mathcal{C}} \frac{1}{V_{\gamma}}
$$

for any $L \geqslant 2$, where

$$
V_{\gamma}=\sum_{N \mathfrak{p} \leqslant L} \frac{\left|\Omega_{\mathfrak{p}} \cap \gamma \Gamma_{\mathfrak{p}}^{s c}\right|}{\left|\Gamma_{\mathfrak{p}}^{s c}\right|}
$$

Proof. With the notation for the auxiliary walks $\left(Y_{\gamma, j}\right)$ previously introduced, and writing

$$
\Omega_{\gamma, \mathfrak{p}}=\Omega_{\mathfrak{p}} \cap \gamma \Gamma_{\mathfrak{p}}^{s c}
$$

the event considered is the disjoint union, over $\gamma \in \mathcal{C}$ and $j \geqslant 0$, of the events

$$
S_{\gamma, j}=\left\{\iota_{n}=j \text { and } \pi_{\mathfrak{p}}\left(Y_{\gamma, j}\right) \notin \Omega_{\gamma, \mathfrak{p}} \text { for } N \mathfrak{p} \leqslant L\right\} .
$$

We have $\iota_{n} \leqslant n$ and hence $S_{\gamma, j}=\emptyset$ for all $j>n$. Moreover, by Lemma 5.6, the probability of the union of all $S_{\gamma, j}$ with $j<\frac{1}{2} \frac{n}{|\mathcal{C}|}$ is at most

$$
\mathbf{P}\left(\iota_{n}<\frac{1}{2} \frac{n}{|\mathcal{C}|}\right) \ll \exp \left(-c_{1} n\right),
$$

for some constant $c_{1}>0$. For others values of $j$, we have

$$
\mathbf{P}\left(S_{\gamma, j}\right) \leqslant \mathbf{P}\left(\pi_{\mathfrak{p}}\left(Y_{\gamma, j}\right) \notin \Omega_{\gamma, \mathfrak{p}} \text { for } N \mathfrak{p} \leqslant L\right) \leqslant\left(1+L^{A} \exp \left(-c_{2} j\right)\right)\left(\sum_{N \mathfrak{p} \leqslant L} \frac{\left|\Omega_{\gamma, \mathfrak{p}}\right|}{\left|\Gamma_{\mathfrak{p}}^{s c}\right|}\right)^{-1}
$$

by Proposition 5.5, and this is

$$
\leqslant\left(1+L^{A} \exp \left(-\frac{c_{2} n}{2|\mathcal{C}|}\right)\right)\left(\sum_{N \mathfrak{p} \leqslant L} \frac{\left|\Omega_{\gamma, \mathfrak{p}}\right|}{\left|\Gamma_{\mathfrak{p}}^{s c}\right|}\right)^{-1}
$$

for some constant $c_{2}>0$.

Summing over the values of $j$ and $\gamma$, we obtain the desired statement, with the constant $c$ given by $\min \left(c_{1}, c_{2} /(2|\mathcal{C}|)\right)$.

Remark 5.8. This result is slightly weaker than the sieve bound for the simply connected case, but it is very close in applications. The intervention of the cosets $\gamma$ in the sieve bound can not be dispensed with in general (i.e., Proposition 5.5 fails if $\Gamma^{s c}$ is replaced with $\Gamma$ ): suppose, say, that $|\mathcal{C}|=2$ with $\Gamma_{\mathfrak{p}}^{s c}$ also of index 2 in $\Gamma_{\mathfrak{p}}$; then, if $\Omega_{\mathfrak{p}}$ is the non-trivial coset of $\Gamma_{\mathfrak{p}}^{s c}$, we have

$$
\mathbf{P}\left(\pi_{\mathfrak{p}}\left(X_{n}\right) \notin \Omega_{\mathfrak{p}} \text { for } N \mathfrak{p} \leqslant L\right) \geqslant \mathbf{P}\left(X_{n} \in \Gamma^{s c}\right)
$$

which typically converges to $\frac{1}{2}$ as $n \rightarrow+\infty$, while an hypothetical estimate like

$$
\left(1+L^{A} \exp (-c n)\right)\left(\sum_{N \mathfrak{p} \leqslant L} \frac{\left|\Omega_{\mathfrak{p}}\right|}{\left|G_{\mathfrak{p}}\right|}\right)^{-1}=2\left(1+L^{A} \exp (-c n)\right) \pi(L)^{-1}
$$

with $c>0$ for this probability would show it to go to zero exponentially fast as $n \rightarrow+\infty$, after selecting $L=\exp (c n / A)$. (On the other hand, in Corollary 5.7, the term involving the non-trivial coset $\Gamma-\Gamma^{s c}$ is the inverse of

$$
\sum_{N \mathfrak{p} \leqslant L} \frac{\left|\Omega_{\mathfrak{p}} \cap\left(\Gamma_{\mathfrak{p}}-\Gamma_{\mathfrak{p}}^{s c}\right)\right|}{\left|\Gamma_{\mathfrak{p}}^{s c}\right|}=0,
$$

so the proposition does work in this context.) 
Remark 5.9. We are considering semisimple groups here because sieving in all arithmetic subgroups of reductive groups is problematic for well-known reasons: if there is a non-trivial central torus $\mathbf{T} \subset$ $Z(\mathbf{G})$, sieving questions might involve unknown issues like the existence of infinitely many Mersenne primes. Moreover, although it is tempting to try to apply once more the strategy described in the discussion following Proposition 5.2, the subgroup $Z(\mathbf{G})$ does not necessarily have finite index in $\mathbf{G}$ (say if $\mathbf{G}=\mathrm{GL}(n)$ and $k$ is a real quadratic field, so that there are infinitely many units), and usually the random walk will not come back infinitely often to each coset.

Remark 5.10. It is very likely that what we have done in this section is valid when $\Gamma$ is simply a finitely generated Zariski-dense subgroup of $\mathbf{G}\left(\mathbf{Z}_{k}\right)$, but not necessarily of finite index (due to recent breakthroughs by Helfgott [He], Bourgain-Gamburd [BG], Breuillard-Green-Tao [BGT] and Pyber-Szabó [PS] playing a key role in the proof by Salehi-Golsefidy-Varjú ([SaV]) that Zariskidense subgroups of arithmetic groups have Property $(\tau)$ with respect to congruence subgroups.)

\section{Splittings FiElds of Elements of Reductive Groups}

In this section, we will prove our main theorem which generalizes Theorem 1.1. Let $\mathbf{G}$ be a connected linear algebraic group defined over a number field $k$. Let $\Gamma \subseteq \mathbf{G}(k)$ be an arithmetic subgroup of $\mathbf{G}$ and let $S$ be a finite symmetric set of generators, such that $(\Gamma, S)$ is balanced (in the sense of the beginning of Section 5). Assume that $\Gamma$ is Zariski-dense in G.

Let $\rho: \mathbf{G} \rightarrow \mathbf{G L}(m)$ be a faithful representation of $\mathbf{G}$ defined over $k$. For each element $g \in \mathbf{G}(k)$, the field $k_{g}$ is defined as the splitting over $k$ of the polynomial $\operatorname{det}(T-\rho(g)) \in k[T]$. From Lemma 2.3(i), we know that $k_{g}$ does not depend on the choice of $\rho$.

As in $\S 5$, let $\left(\xi_{n}\right)$ be a sequence of independent, identically distributed, random variables taking values in $S$ such that $\mathbf{P}\left(\xi_{n}=s\right)=\mathbf{P}\left(\xi_{n}=s^{-1}\right)>0$ for all $s \in S$. The sequence $\left(X_{n}\right)$ defined recursively by

$$
X_{0}=1 \in \Gamma, \quad X_{n+1}=X_{n} \xi_{n+1},
$$

gives a random walk on $\Gamma$.

For a reductive group $\mathbf{G}$, we defined in $\S 2.2$ an extension $k_{\mathbf{G}} / k$ and groups $W(\mathbf{G})$ and $\Pi(\mathbf{G})$.

When $\mathbf{G}$ is not reductive, we set $k_{\mathbf{G}}:=k_{\mathbf{G} / R_{u}(\mathbf{G})}$ and make the ad hoc definitions $W(\mathbf{G}):=$ $W\left(\mathbf{G} / R_{u}(\mathbf{G})\right)$ and $\Pi(\mathbf{G}):=\Pi\left(\mathbf{G} / R_{u}(\mathbf{G})\right)$, where $R_{u}(\mathbf{G})$ is the unipotent radical of $\mathbf{G}$.

Theorem 6.1. Fix notation and assumptions as above.

(i) We have

$$
\lim _{n \rightarrow \infty} \mathbf{P}\left(\operatorname{Gal}\left(k_{X_{n}} / k\right) \cong \Pi(\mathbf{G})\right)=1 .
$$

(ii) If $\mathbf{G}$ is semisimple, then there exists a constant $c>1$ such that

$$
\mathbf{P}\left(\operatorname{Gal}\left(k_{X_{n}} / k\right) \cong \Pi(\mathbf{G})\right)=1+O\left(c^{-n}\right)
$$

for all $n \geqslant 1$.

(iii) There exists a constant $c>1$ such that

$$
\mathbf{P}\left(\operatorname{Gal}\left(k_{\mathbf{G}} k_{X_{n}} / k_{\mathbf{G}}\right) \cong W(\mathbf{G})\right)=1+O\left(c^{-n}\right)
$$

for all $n \geqslant 1$.

The constants $c$ and the implicit constants depend only on the group $\mathbf{G}$, the generating set $S$, and the distribution of the $\xi_{n}$.

Theorem 1.1(i) is a consequence of Lemma 2.4(i). We obtain the remaining parts of Theorem 1.1 from Theorem 6.1 by taking $p(s)=|S|^{-1}$ for all $s \in S$, which then implies by definition that

$$
\mathbf{P}\left(X_{n} \in A\right)=\frac{1}{|S|^{n}}\left|\left\{w=\left(s_{1}, \ldots, s_{n}\right) \in S^{n} \mid s_{1} \cdots s_{n} \in A\right\}\right|
$$


for any set $A \subset \Gamma$.

Our first lemma is a version, in our context, of a "non-concentration" estimate on subvarieties.

Lemma 6.2. Keep the set-up as above and let $Y \subsetneq \mathbf{G}$ be a closed subvariety that is stable under conjugation by $\mathbf{G}$.

(i) We have $\lim _{n \rightarrow \infty} \mathbf{P}\left(X_{n} \in Y(k)\right)=0$.

(ii) If $\mathbf{G}$ is semisimple, then there exists a constant $c>1$ such that

$$
\mathbf{P}\left(X_{n} \in Y(k)\right)=O\left(c^{-n}\right) .
$$

The constant $c$ and the implicit constant depend only on the group $\mathbf{G}$, the generating set $S$, the distribution of the $\xi_{n}$, and $Y$.

Proof. We start with the proof of (ii), which is more precise. Choose a model $\mathcal{G}$ over $\mathbf{Z}_{k}\left[R^{-1}\right]$ of $\mathbf{G}$ where $R$ is a finite set of maximal ideals of $\mathbf{Z}_{k}$. Since $\Gamma$ is finitely generated, we can choose $R$ so that $\Gamma \subseteq \mathcal{G}\left(\mathbf{Z}_{k}\left[R^{-1}\right]\right)$. For $\mathfrak{p} \notin R$, let $\pi_{\mathfrak{p}}: \Gamma \rightarrow \mathcal{G}\left(\mathbf{F}_{\mathfrak{p}}\right)$ be the reduction modulo $\mathfrak{p}$ map. Let $\mathcal{Y}$ be the Zariski closure of $Y$ in $\mathcal{G}$, and for each $\mathfrak{p} \notin R$, define the set

$$
\Omega_{\mathfrak{p}}=\mathcal{G}\left(\mathbf{F}_{\mathfrak{p}}\right)-\mathcal{Y}\left(\mathbf{F}_{\mathfrak{p}}\right) .
$$

Our assumption that $Y$ is stable under conjugation by $\mathbf{G}$ implies that $\Omega_{\mathfrak{p}}$ is stable under conjugation by $\mathcal{G}\left(\mathbf{F}_{\mathfrak{p}}\right)$. If $X_{n} \in \Gamma$ belongs to $Y(k)$, then $\pi_{\mathfrak{p}}\left(X_{n}\right) \notin \Omega_{\mathfrak{p}}$ for all $\mathfrak{p} \notin R$. Thus for any $L \geqslant 2$, we have

$$
\mathbf{P}\left(X_{n} \in Y(k)\right) \leqslant \mathbf{P}\left(\pi_{\mathfrak{p}}\left(X_{n}\right) \notin \Omega_{\mathfrak{p}} \text { for all } \mathfrak{p} \notin R \text { with } N \mathfrak{p} \leqslant L\right) .
$$

We are now in a position to apply (5.5) to derive the upper bound

$$
\mathbf{P}\left(X_{n} \in Y(k)\right) \leqslant B e^{-c n}+n\left(1+L^{A} e^{-c n}\right) \sum_{\gamma \in \mathcal{C}} \frac{1}{V_{\gamma}},
$$

where $A \geqslant 0, B \geqslant 0$ and $c>0$ are constants depending only on $k, \mathcal{C}=\Gamma^{s c} \backslash \Gamma$, and

$$
V_{\gamma}:=\sum_{\substack{N \mathfrak{p} \leqslant L \\ \mathfrak{p} \notin R}} \frac{\left|\Omega_{\mathfrak{p}} \cap \gamma \Gamma_{\mathfrak{p}}^{s c}\right|}{\left|\Gamma_{\mathfrak{p}}^{s c}\right|}=\sum_{\substack{N \mathfrak{p} \leqslant L \\ \mathfrak{p} \notin R}}\left(1-\frac{\left|\mathcal{Y}\left(\mathbf{F}_{\mathfrak{p}}\right) \cap \gamma \Gamma_{\mathfrak{p}}^{s c}\right|}{\left|\Gamma_{\mathfrak{p}}^{s c}\right|}\right) .
$$

Since $Y$ has smaller dimension that $\mathbf{G}$, the Lang-Weil bounds (see, e.g., [Ka, p. 628]) imply that

$$
\left|\mathcal{Y}\left(\mathbf{F}_{\mathfrak{p}}\right)\right| /\left|\mathcal{G}\left(\mathbf{F}_{\mathfrak{p}}\right)\right|=O(1 / N(\mathfrak{p}))
$$

This, together with $\left[\mathcal{G}\left(\mathbf{F}_{\mathfrak{p}}\right): \Gamma_{\mathfrak{p}}^{s c}\right] \ll 1$, gives

$$
V_{\gamma} \gg \sum_{\substack{N \mathfrak{p} \leqslant L \\ \mathfrak{p} \notin R}}\left(1+O\left(N(\mathfrak{p})^{-1}\right)\right) \gg L / \log L,
$$

where the last inequality holds for all $L$ sufficiently large. Thus

$$
\mathbf{P}\left(X_{n} \in Y(k)\right) \ll e^{-c n}+\frac{n\left(1+L^{A} e^{-c n}\right)}{L}(\log L)
$$

for $L$ sufficiently large. Setting $L=\exp \left(n c_{1} / A\right)$ with $c_{1}<c$ a positive number, gives

$$
\mathbf{P}\left(X_{n} \in Y(k)\right) \ll e^{-c n}+n^{2} e^{-c_{1} n / A}+n^{2} e^{-\left(c-c_{1}\right) n} e^{-c_{1} n / A}
$$

for $n$ large enough. So there is a $c_{2}>0$ such that $\mathbf{P}\left(X_{n} \in Y(k)\right) \ll e^{-c_{2} n}=\left(e^{-c_{2}}\right)^{n}$ for all $n \geqslant 1$.

Now we come to (i). Let $\mathbf{G}_{1}$ be the derived group of $\mathbf{G}, \mathbf{T}$ the connected component of the center of $\mathbf{G}$, so that we have a surjective product map

$$
\mathbf{G}_{1} \times \mathbf{T} \rightarrow \mathbf{G}
$$


with finite fibers. We are going to reduce the question to one on $\mathbf{G}_{1} \times \mathbf{T}$. For this, we observe that there exists a fixed number field $k_{S} / k$ and elements $\left(x_{s}, z_{s}\right) \in\left(\mathbf{G}_{1} \times \mathbf{T}\right)\left(k_{S}\right)$ for all $s \in S$ such that

$$
s=x_{s} z_{s}
$$

for all $s$. We can then write $X_{n}=Z_{n} \tilde{X}_{n}$ with random variables

$$
\tilde{X}_{n}=x_{\xi_{1}} \cdots x_{\xi_{n}}, \quad Z_{n}=z_{\xi_{1}} \cdots z_{\xi_{n}}
$$

taking values in $\left(\mathbf{G}_{1} \times \mathbf{T}\right)\left(k_{S}\right)$, which form random walks on (subgroups of) $\mathbf{G}_{1}\left(k_{S}\right)$ and $\mathbf{T}\left(k_{S}\right)$.

Now let $\tilde{Y}$ be the inverse image of $Y$ in $\mathbf{G}_{1} \times \mathbf{T}$. If the projection of $\tilde{Y}$ on $\mathbf{G}_{1}$ is not dense, say it is contained in a proper (conjugacy-invariant) subvariety $Y_{1} \subset \mathbf{G}_{1}$, the condition $X_{n} \in Y(k)$ implies $\tilde{X}_{n} \in Y_{1}\left(k_{S}\right)$, which occurs with probability tending to 0 by applying (ii) (with $k$ replaced by $k_{S}$ ). Otherwise, the projection of $\tilde{Y}$ on the torus $\mathbf{T}$ must be contained in a proper subvariety, say $Y_{2}$, and the condition $X_{n} \in Y(k)$ implies $Z_{n} \in Y_{2}\left(k_{S}\right)$. In fact, $Z_{n}$ lies in the finite rank abelian group generated by the $z_{s}$ in $\mathbf{T}$ (in fact, $\mathbf{T}\left(k_{S}\right)$ is itself of finite rank, by the generalized Dirichlet unit theorem, see, e.g., [PR, Cor. 1, p. 209]), and we are therefore reduced to a question that can be handled by more classical sieve methods, for instance by the large sieve on $\mathbf{Z}^{n}$, as described in $[\mathrm{K}, \S 4.2]$. Using reductions modulo primes (of $k_{S}$ ) and the Lang-Weil estimate for $Y_{2}$ to estimate the number of permitted residue classes for $Y_{2}\left(k_{S}\right)$, we obtain the qualitative estimate (i).

Remark 6.3. We used the sieve result of the previous section, but one could also deal with this by selecting a single well-chosen prime ideal. We also see clearly that (i) could be replaced, with some work, by an estimate of quantitative decay, which would however only be of the type $n^{-c}$ for some fixed $c>0$.

The following proposition, which is given for semisimple groups, will be key in the proof of Theorem 6.1. The proof follows the same basic principle as earlier works using the large sieve to study probabilistic Galois theory: the sieve implies that Frobenius elements in $\operatorname{Gal}\left(k_{X_{n}} / k\right)$ can be found (with very high probability) that map to any given conjugacy class of $W(\mathbf{G})$ under the injective homomorphism of Section 4, and we can then use the well-known lemma of Jordan according to which, in a finite group, no proper subgroup contains elements of all conjugacy classes.

Proposition 6.4. Fix notation and assumptions as above, and assume that $\mathbf{G}$ is semisimple. Let $K \subseteq \bar{k}$ be a finite extension of $k_{\mathbf{G}}$. Then there exists a constant $c>1$ such that

$$
\mathbf{P}\left(\operatorname{Gal}\left(K k_{X_{n}} / K\right) \cong W(\mathbf{G})\right)=1+O\left(c^{-n}\right) .
$$

The constant $c$ and the implicit constant depend only on the group $\mathbf{G}$, the generating set $S$, the distribution of the $\xi_{n}$, and the field $K$.

Proof. Fix a maximal torus $\mathbf{T}_{0}$ of $\mathbf{G}$. By Lemma 2.4, the group $\operatorname{Gal}\left(k_{\mathbf{G}} k_{X_{n}} / k_{\mathbf{G}}\right)$ is isomorphic to a subquotient of $W(\mathbf{G})$. So without loss of generality, we may extend $K$ so that $\mathbf{T}_{0, K}$ is split. Choose a semisimple group scheme $\mathcal{G}$ over $\mathbf{Z}_{k}\left[R^{-1}\right]$ whose generic fiber is $\mathbf{G}$ where $R$ is a finite set of maximal ideals of $\mathbf{Z}_{k}$. Let $\mathcal{T}_{0}$ be the Zariski closure of $\mathbf{T}_{0}$ in $\mathcal{G}$. By taking $R$ large enough, we may assume that $\mathcal{T}_{0}$ is a maximal torus of $\mathcal{G}$ and $\Gamma \subseteq \mathcal{G}\left(\mathbf{Z}_{k}\left[R^{-1}\right]\right)$. For $\mathfrak{p} \notin R$, let $\pi_{\mathfrak{p}}: \Gamma \rightarrow \mathcal{G}\left(\mathbf{F}_{\mathfrak{p}}\right)$ be the homomorphism of reduction modulo $\mathfrak{p}$.

Let $\mathcal{P}$ be the set of maximal ideals $\mathfrak{p} \notin R$ of $\mathbf{Z}_{k}$ that split completely in $K$. For $\mathfrak{p} \in \mathcal{P}$, the tori $\mathcal{T}_{0, k_{\mathfrak{p}}}$ and $\mathcal{T}_{0, \mathbf{F}_{\mathfrak{p}}}$ are split. The set $\mathcal{P}$ has positive natural density, by the Chebotarev density theorem (see, e.g., [IK, p. 143].) For each $\mathfrak{p} \in \mathcal{P}$, fix an embedding $\bar{k} \hookrightarrow \bar{k}_{\mathfrak{p}}$ which is the identity map on $k$. Let $W(\mathbf{G})^{\sharp} \leftrightarrow W\left(\mathcal{G}_{\mathbf{F}_{\mathfrak{p}}}\right)^{\sharp}$ be the bijection (3.3); we will use it as an identification.

For $\mathfrak{p} \in \mathcal{P}$, we can define a map

$$
\theta_{\mathfrak{p}}: \mathcal{G}\left(\mathbf{F}_{\mathfrak{p}}\right)_{s r} \rightarrow W\left(\mathcal{G}_{\mathbf{F}_{\mathfrak{p}}}\right)^{\sharp}=W(\mathbf{G})^{\sharp}
$$


as in $\S 4$. Fix a conjugacy class $C \in W(\mathbf{G})^{\sharp}$. For $\mathfrak{p} \in \mathcal{P}$, define the set

$$
\Omega_{\mathfrak{p}}:=\left\{g \in \mathcal{G}\left(\mathbf{F}_{\mathfrak{p}}\right)_{s r}: \theta_{\mathfrak{p}}(g)=C\right\} .
$$

Let $\mathbf{G}(k)_{s r}$ be the set of $g \in \mathbf{G}(k)$ that are semisimple and regular in $\mathbf{G}$. For $X_{n} \in \mathbf{G}(k)_{s r}$, we have defined a representation $\varphi_{X_{n}}: \operatorname{Gal}(\bar{k} / k) \rightarrow \Pi(\mathbf{G})$ that is uniquely defined up to conjugation by an element of $W(\mathbf{G})$. Fix a prime $\mathfrak{p} \in \mathcal{P}$ such that $\pi_{\mathfrak{p}}\left(X_{n}\right) \in \Omega_{\mathfrak{p}}$. By Proposition 3.2, $\varphi_{X_{n}}$ is unramified at $\mathfrak{p}$ and the conjugacy class of the Frobenius automorphism Frob $\mathfrak{p}_{\mathfrak{p}}$ at $\mathfrak{p}$ is $C$, i.e., $\varphi_{X_{n}}\left(\right.$ Frob $\left._{\mathfrak{p}}\right)=C$.

Since $\mathfrak{p}$ splits completely in $K$, we deduce that

$$
\varphi_{X_{n}}(\operatorname{Gal}(\bar{k} / K)) \cap C \neq \emptyset,
$$

and therefore, we have an upper bound

$$
\begin{aligned}
\mathbf{P}\left(X_{n} \in \mathbf{G}(k)_{s r} \text { and } \varphi_{X_{n}}(\operatorname{Gal}(\bar{k} / K)) \cap C=\emptyset\right) \\
\quad \leqslant \mathbf{P}\left(\pi_{\mathfrak{p}}\left(X_{n}\right) \notin \Omega_{\mathfrak{p}} \text { for all } \mathfrak{p} \in \mathcal{P} \text { with } N \mathfrak{p} \leqslant L\right),
\end{aligned}
$$

where the last probability is amenable to sieve. Specifically, applying (5.5), we derive the upper bound

$$
\mathbf{P}\left(\pi_{\mathfrak{p}}\left(X_{n}\right) \notin \Omega_{\mathfrak{p}} \text { for all } \mathfrak{p} \in \mathcal{P} \text { with } N \mathfrak{p} \leqslant L\right) \leqslant B e^{-c n}+n\left(1+L^{A} e^{-c n}\right) \sum_{\gamma \in \mathcal{C}} \frac{1}{V_{\gamma}},
$$

where $A \geqslant 0, B \geqslant 0$ and $c>0$ are constants depending only on $k, \mathcal{C}=\Gamma^{s c} \backslash \Gamma$, and

$$
V_{\gamma}:=\sum_{\substack{N \mathfrak{p} \leqslant L \\ \mathfrak{p} \in \mathcal{P}}} \frac{\left|\Omega_{\mathfrak{p}} \cap \gamma \Gamma_{\mathfrak{p}}^{s c}\right|}{\left|\Gamma_{\mathfrak{p}}^{s c}\right|}
$$

By Proposition 4.6, we have

$$
\frac{\left|\Omega_{\mathfrak{p}} \cap \gamma \Gamma_{\mathfrak{p}}^{s c}\right|}{\left|\Gamma_{\mathfrak{p}}^{s c}\right|}=\frac{|C|}{|W(\mathbf{G})|}+O\left(N(\mathfrak{p})^{-1}\right)
$$

for all $\gamma \in \mathcal{C}$ and $\mathfrak{p} \in \mathcal{P}$, where the implicit constant does not depend on $\mathfrak{p}$. This implies that

$$
V_{\gamma} \gg \sum_{\substack{N \mathfrak{p} \leqslant L \\ \mathfrak{p} \in \mathcal{P}}}\left(\frac{|C|}{|W(\mathbf{G})|}+O\left(N(\mathfrak{p})^{-1}\right)\right) \gg L / \log L
$$

where the last inequality holds for all $L$ sufficiently large (since $\mathcal{P}$ has positive density). Therefore,

$$
\mathbf{P}\left(\pi_{\mathfrak{p}}\left(X_{n}\right) \notin \Omega_{\mathfrak{p}} \text { for all } \mathfrak{p} \in \mathcal{P} \text { with } N \mathfrak{p} \leqslant L\right) \ll e^{-c n}+\frac{n\left(1+L^{A} e^{-c n}\right)}{L}(\log L) .
$$

for all $L$ sufficiently large. As at the end of the proof of Lemma 6.2, we obtain

$$
\mathbf{P}\left(\pi_{\mathfrak{p}}\left(X_{n}\right) \notin \Omega_{\mathfrak{p}} \text { for all } \mathfrak{p} \in \mathcal{P} \text { with } N \mathfrak{p} \leqslant L\right) \ll c^{-n}
$$

for some $c>1$.

So from (6.1), we find that

$$
\mathbf{P}\left(X_{n} \in \mathbf{G}(k)_{s r} \text { and } \varphi_{X_{n}}(\operatorname{Gal}(\bar{k} / K)) \cap C=\emptyset\right) \ll c^{-n}
$$


for some constant $c>1$, which we may assume holds for all $C \in W(\mathbf{G})^{\sharp}$. By Jordan's lemma, no proper subgroup of $W(\mathbf{G})$ intersects every conjugacy class of $W(\mathbf{G})$. Therefore,

$$
\begin{aligned}
& \mathbf{P}\left(X_{n} \in \mathbf{G}(k)_{s r} \text { and } \varphi_{X_{n}}(\operatorname{Gal}(\bar{k} / K)) \neq W(\mathbf{G})\right) \leqslant \\
& \qquad \sum_{C \in W(\mathbf{G})^{\sharp}} \mathbf{P}\left(X_{n} \in \mathbf{G}(k)_{s r} \text { and } \varphi_{X_{n}}(\operatorname{Gal}(\bar{k} / K)) \cap C=\emptyset\right) \ll c^{-n} .
\end{aligned}
$$

Now let $Y$ be the subvariety of $\mathbf{G}$ from Lemma 2.4(iii). By Lemma 6.2, we have

$$
\mathbf{P}\left(X_{n} \in Y(k)\right) \ll c^{-n},
$$

(after possibly increasing $c>1$ ). If $X_{n} \notin Y(k)$ and $\varphi_{X_{n}}(\mathrm{Gal}(\bar{k} / K))=W(\mathbf{G})$, then $X_{n}$ is regular and semisimple in $\mathbf{G}$ and $\operatorname{Gal}\left(K k_{X_{n}} / K\right) \cong W(\mathbf{G})$. Therefore,

$$
\mathbf{P}\left(\operatorname{Gal}\left(K k_{X_{n}} / K\right) \nRightarrow W(\mathbf{G})\right) \ll c^{-n}
$$

for some constant $c>1$.

6.1. Proof of Theorem 6.1. We first consider the case where $\mathbf{G}$ is reductive. Let $R(\mathbf{G})$ be the radical of $\mathbf{G}$. Since $\mathbf{G}$ is reductive, $R(\mathbf{G})$ is the connected component of the center of $\mathbf{G}$. The quotient $\mathbf{G}^{\prime}:=\mathbf{G} / R(\mathbf{G})$ is defined over $k$ and is semisimple. Let $\pi: \mathbf{G} \rightarrow \mathbf{G}^{\prime}$ be the quotient homomorphism.

We now consider $\Gamma^{\prime} \subseteq \mathbf{G}^{\prime}(k)$, the image of $\Gamma$ under $\pi$, and the generating set $S^{\prime}$ which is the image of $S$. The pair $\left(\Gamma^{\prime}, S^{\prime}\right)$ is still balanced. The group $\Gamma^{\prime}$ is Zariski dense in $\mathbf{G}^{\prime}$ since $\Gamma$ is Zariski dense in $\mathbf{G}$ and $\pi$ is surjective; again because $\pi$ is surjective, and $\Gamma$ is arithmetic, we find that $\Gamma^{\prime}$ is an arithmetic subgroup of $\mathbf{G}^{\prime}$.

To the random walk $\left(X_{n}\right)$ on $\Gamma$, we can associate the random walk $\left(X_{n}^{\prime}\right)$ on $\Gamma^{\prime}$ where $X_{n}^{\prime}=\pi\left(X_{n}\right)$. It is a left-invariant random walk defined by the sequence of steps $\left(\xi_{n}^{\prime}\right)$ where $\xi_{n}^{\prime}=\pi\left(\xi_{n}\right)$. Each $\xi_{n}^{\prime}$ takes values in the symmetric generating system $S^{\prime}$ of $\Gamma^{\prime}$ and has distribution

$$
\mathbf{P}\left(\xi_{n}^{\prime}=s^{\prime}\right)=\sum_{s \in S, \pi(s)=s^{\prime}} p(s)
$$

for $s^{\prime} \in S^{\prime}$. We have $\mathbf{P}\left(\xi_{n}^{\prime}=s^{\prime}\right)=\mathbf{P}\left(\xi_{n}^{\prime}=\left(s^{\prime}\right)^{-1}\right)>0$ for all $s^{\prime} \in S^{\prime}$, and the random variables $\left(\xi_{n}^{\prime}\right)$ are independent and identically distributed.

Lemma 6.5. We have $k_{X_{n}^{\prime}} \subseteq k_{X_{n}}$.

Proof. More generally, we claim that $k_{\pi(g)} \subseteq k_{g}$ for all $g \in \mathbf{G}(k)$. Without loss of generality, we may assume that $g$, and hence $\pi(g)$, is semisimple. Let $\mathbf{T}$ be a maximal torus of $\mathbf{G}$ containing $g$. The torus $\mathbf{T}^{\prime}:=\mathbf{T} / R(\mathbf{G})$ is then a maximal torus of $\mathbf{G}^{\prime}$ which contains $\pi(g)$. The homomorphism $X\left(\mathbf{T}^{\prime}\right) \rightarrow X(\mathbf{T}), \chi^{\prime} \mapsto \chi^{\prime} \circ \pi$, gives an inclusion

$$
\left\{\chi^{\prime}(\pi(g)): \chi^{\prime} \in X\left(\mathbf{T}^{\prime}\right)\right\} \subseteq\{\chi(g): \chi \in X(\mathbf{T})\} .
$$

By Lemma 2.4(ii), we deduce that $k_{\pi(g)} \subseteq k_{g}$.

Fix a finite extension $K$ of $k$ that contains $k_{\mathbf{G}}$ and $k_{\mathbf{G}^{\prime}}$. Suppose that $\operatorname{Gal}\left(K k_{X_{n}^{\prime}} / K\right) \cong W\left(\mathbf{G}^{\prime}\right)$. By Lemma 6.5, we have $\left|\operatorname{Gal}\left(K k_{X_{n}} / K\right)\right| \geqslant\left|W\left(\mathbf{G}^{\prime}\right)\right|$. Since $\mathbf{G}$ and $\mathbf{G}^{\prime}$ have isomorphic Weyl groups, we have $\left|\operatorname{Gal}\left(K k_{X_{n}} / K\right)\right| \geqslant|W(\mathbf{G})|$. By Lemma 2.4(i), $\operatorname{Gal}\left(K k_{X_{n}} / K\right)$ is isomorphic to a subquotient of $W(\mathbf{G})$, so by cardinality considerations we find that $\operatorname{Gal}\left(K k_{X_{n}} / K\right) \cong W(\mathbf{G})$.

Therefore,

$$
\mathbf{P}\left(\operatorname{Gal}\left(K k_{X_{n}^{\prime}} / K\right) \cong W\left(\mathbf{G}^{\prime}\right)\right) \leqslant \mathbf{P}\left(\operatorname{Gal}\left(K k_{X_{n}} / K\right) \cong W(\mathbf{G})\right)
$$

Since $\mathbf{G}^{\prime}$ is semisimple, Proposition 6.4 implies that

$$
\mathbf{P}\left(\operatorname{Gal}\left(K k_{X_{n}^{\prime}} / K\right) \cong W\left(\mathbf{G}^{\prime}\right)\right)=1+O\left(c^{-n}\right)
$$


for some constant $c>1$, and therefore

$$
\mathbf{P}\left(\operatorname{Gal}\left(K k_{X_{n}} / K\right) \cong W(\mathbf{G})\right)=1+O\left(c^{-n}\right) .
$$

Since $\operatorname{Gal}\left(k_{\mathbf{G}} k_{X_{n}} / k_{\mathbf{G}}\right)$ is always isomorphic to a subquotient of $W(\mathbf{G})$ by Lemma 2.4(i), we deduce that

$$
\mathbf{P}\left(\operatorname{Gal}\left(k_{\mathbf{G}} k_{X_{n}} / k_{\mathbf{G}}\right) \cong W(\mathbf{G})\right)=1+O\left(c^{-n}\right),
$$

and this completes the proof of (iii) in the reductive case.

Let $Y$ be the subvariety of $\mathbf{G}$ from Lemma 2.4(iii). Fix $g \in \mathbf{G}(k)-Y(k)$ such that

$$
\operatorname{Gal}\left(k_{\mathbf{G}} k_{g} / k_{\mathbf{G}}\right) \cong W(\mathbf{G})
$$

We claim that $\operatorname{Gal}\left(k_{g} / k\right) \cong \Pi(\mathbf{G})$. Since $g \notin Y(k), g$ is contained in a unique maximal torus $\mathbf{T}$ of $\mathbf{G}$ and $k_{g}=k_{\mathbf{T}}$. It thus suffices to show that $\operatorname{Gal}\left(k_{\mathbf{T}} / k\right) \cong \Pi(\mathbf{G})$. The homomorphism

$$
\operatorname{Gal}\left(k_{\mathbf{T}} / k\right) \stackrel{\varphi_{\mathbf{T}}}{\longrightarrow} \Pi(\mathbf{G}) \rightarrow \Pi(\mathbf{G}) / W(\mathbf{G})
$$

is surjective and $\varphi_{\mathbf{T}}\left(\operatorname{Gal}\left(k_{T} / k_{\mathbf{G}}\right)\right) \subseteq W(\mathbf{G})$, so it suffices to show that $\operatorname{Gal}\left(k_{T} / k_{\mathbf{G}}\right) \cong W(\mathbf{G})$. But since $k_{\mathbf{T}} \supseteq k_{\mathbf{G}}$, we have $\operatorname{Gal}\left(k_{\mathbf{T}} / k_{\mathbf{G}}\right)=\operatorname{Gal}\left(k_{\mathbf{G}} k_{g} / k_{\mathbf{G}}\right) \cong W(\mathbf{G})$ as desired. Therefore

$$
\mathbf{P}\left(\operatorname{Gal}\left(k_{X_{n}} / k\right) \neq \Pi(\mathbf{G})\right) \leqslant \mathbf{P}\left(\operatorname{Gal}\left(k_{\mathbf{G}} k_{X_{n}} / k_{\mathbf{G}}\right) \neq W(\mathbf{G})\right)+\mathbf{P}\left(X_{n} \in Y(k)\right) .
$$

By part (iii), which we have already proved, we have

$$
\mathbf{P}\left(\operatorname{Gal}\left(k_{X_{n}} / k\right) \neq \Pi(\mathbf{G})\right) \ll c^{-n}+\mathbf{P}\left(X_{n} \in Y(k)\right)
$$

for some constant $c>1$. Part (i) and (ii) in the reductive case then follow immediately from Lemma 6.2.

Finally, we consider the case where $\mathbf{G}$ is not reductive. The quotient $\mathbf{G}^{\prime}:=\mathbf{G} / R_{u}(\mathbf{G})$ is defined over $k$ and is reductive. Let $\pi: \mathbf{G} \rightarrow \mathbf{G}^{\prime}$ be the quotient homomorphism. As above, we can consider the arithmetic subgroup $\Gamma^{\prime}:=\pi(\Gamma)$ of $\mathbf{G}^{\prime}(k)$ and the related random walk $\left(X_{n}^{\prime}\right)$ on $\Gamma^{\prime}$ where $X_{n}^{\prime}=\pi\left(X_{n}\right)$. By Lemma 2.3, we have $k_{X_{n}}=k_{X_{n}^{\prime}}$. The non reductive case then follows directly from the reductive case.

\section{COMments ON OTHER APPROACHES}

One may wonder about our use of random walks to quantify the maximality principle for splitting fields, and it is natural to see why it is interesting, and what other approaches to "random" elements are possible.

These are essentially of two kinds: one could try to prove upper bounds for the density

$$
\frac{\mid\{g \in \Gamma \mid\|\iota(g)\| \leqslant X \text { and } \operatorname{det}(T-\iota(g)) \text { has "small" Galois group }\} \mid}{|\{g \in \Gamma \mid\|\iota(g)\| \leqslant X\}|},
$$

as $X$ grows, where $\iota$ denotes a fixed faithful representation of $\mathbf{G}$ into some $G L(n)$ and $\|g\|$ is (say) the Hilbert-Schmidt norm on $\mathrm{GL}(n, \mathbf{C})$. Or one could still use the system of generators $S$ but try to bound

$$
\frac{\mid\left\{g \in \Gamma \mid \ell_{S}(g) \leqslant X \text { and } \operatorname{det}(T-\iota(g)) \text { has "small" Galois group }\right\} \mid}{\left|\left\{g \in \Gamma \mid \ell_{S}(g) \leqslant X\right\}\right|},
$$

where $\ell_{S}(g)$ is the combinatorial distance on $\Gamma$ defined by $S$. The sieve techniques can potentially extend to these situations, but one needs to know good equidistribution properties for reduction modulo primes in these two types of balls, uniformly and quantitatively. The uniformity will ultimately depend on the spectral gap property of $\Gamma$ (i.e., on Property $(\tau)$ ), but due to the relations in the group, it is not so easy to derive from it the required equidistribution, in the combinatorial case (one would need to do it in each coset of $\Gamma^{s c}$, of course). In the archimedean case, this has very recently been implemented along these lines by Gorodnik and Nevo [GN1], using their deep ergodic-theoretic equidistribution results [GN2]. 
Moreover, in comparison with these two other approaches, random walks have one interesting feature: they lend themselves readily to concrete computations, and in this respect can be pretty efficient. This is illustrated, in the earlier paper [JKZ], by the fairly small size of the polynomial $P$ with Galois group $W\left(\mathbf{E}_{8}\right)$ that we obtained, especially if the corresponding element of $\mathbf{E}_{8}(\mathbf{Q})$ is expressed as a product of standard Steinberg generators $x_{1}, \ldots, x_{8}$ : we have simply

$$
P=\operatorname{det}\left(T-\operatorname{Ad}\left(x_{1} \cdots x_{8} x_{1}^{-1} \cdots x_{8}^{-1}\right)\right) /(T-1)^{8} \in \mathbf{Z}[T] .
$$

In other words, the complexity of the polynomial (if not of the splitting field, in terms of usual algebraic invariants such as the discriminant of the ring of integers, which is difficult to control) is fairly directly related to the length of the walk.

Another point is that random walks enable us to state some corollaries, and ask some questions, which do not make sense for other meanings of "random" elements. For instance, given the random walk $\left(X_{n}\right)$ as in Theorem 6.1, it follows (in the semisimple case) from the Borel-Cantelli Lemma that, almost surely, there are only finitely many $n$ for which $\operatorname{Gal}\left(k_{X_{n}} / k\right)$ is not isomorphic to $W(\mathbf{G})$. We can then ask how the random variables

$$
\begin{aligned}
\tau & =\min \left\{n \geqslant 1 \mid \operatorname{Gal}\left(k_{X_{n}} / k\right)=W(\mathbf{G})\right\}, \\
\tau^{*} & =\max \left\{n \geqslant 1 \mid \operatorname{Gal}\left(k_{X_{n}} / k\right) \neq W(\mathbf{G})\right\}
\end{aligned}
$$

are distributed?

\section{REFERENCES}

[BW] R.N. Bhattacharya and E.C. Waymire: Stochastic processes with applications, Wiley Series in Probability and Mathematical Statistics, Wiley, 1990. 5, 5, 5

[Bo] A. Borel: Linear algebraic groups, 2nd edition, GTM 126, Springer 1991. 2.2, 2.3, 4.1

[BG] J. Bourgain and A. Gamburd: Uniform expansion bounds for Cayley graphs of $\mathrm{SL}_{2}\left(\mathbf{F}_{p}\right)$, Ann. of Math. (2) 167 (2008), 625-642. 5.10

[BGT] E. Breuillard, B. Green and T.Tao: Linear approximate groups, preprint (2010), arXiv:1005.1881 5.10

[Ca1] R.W. Carter: Finite groups of Lie type. Conjugacy classes and complex characters. Pure and Applied Mathematics. A Wiley-Interscience Publication. John Wiley \& Sons, Inc., New York, 1985. 4.2, 4.1, 4.1, 4.1, 4.1

[Ca2] R.W. Carter: Semisimple conjugacy classes and classes in the Weyl group, Journal of Algebra 260 (2003), 99-110. 1, 4.3

[Cl] L. Clozel: Démonstration de la conjecture $\tau$, Invent. math. 151 (2003), 297-328. 5

[D] M. Demazure: Schémas en groupes réductifs, Bull. Soc. Math. France 93, (1965), 369-413. 3

[F] J. Fulman: Applications of the Brauer complex: card shuffling, permutation statistics, and dynamical systems, Journal of Algebra 243 (2001), 96-122. 1, 4.3

[GN1] A. Gorodnik and A. Nevo: Splitting fields of elements in arithmetic groups, preprint (2011). 7

[GN2] A. Gorodnik and A. Nevo: The ergodic theory of lattice subgroups, Annals of Math. Studies 172, Princeton Univ. Press, 2010. 7

[He] H. Helfgott: Growth and generation in $S L_{2}(\mathbf{Z} / p \mathbf{Z})$, Ann. of Math. 167 (2008), 601-623. 5.10

[HP] E. Hrushovski and A. Pillay: Definable subgroups of algebraic groups over finite fields, J. Reine angew. Math 462 (1995), 69-91. 5

[IK] H. Iwaniec and E. Kowalski: Analytic number theory, American Mathematical Society Colloquium Publications, 53. AMS, Providence, RI, 2004. 6

[JKZ] F. Jouve, E. Kowalski and D. Zywina: An explicit integral polynomial whose splitting field has Galois group $W\left(\mathbf{E}_{8}\right)$, J. Théor. Nombres Bordeaux 20 (2008), 761-782. 1, 7

[J] F. Jouve: The large sieve and random walks on left cosets of arithmetic groups, Comment. Math. Helv. 85 (2010), 647-704. 5, 5, 5, 5

[Ka] N. M. Katz: Factoring polynomials in finite fields: an application of Lang-Weil to a problem in graph theory. Math. Ann. 286 (1990), no. 4, 625-637. 6

[K] E. Kowalski: The large sieve and its applications: arithmetic geometry, random walks, discrete groups, Cambridge Univ. Tracts 175 (2008). 1.2, 5, 5.1, 5, 5, 5, 6

[L] P. Lezaud: Chernoff-type bound for finite Markov chains, Annals Appl. Prob. 8 (1998), 849-867. 5 
[MVW] C.R. Matthews, L.N. Vaserstein and B. Weisfeiler: Congruence properties of Zariski-dense subgroups, Proc. London Math. Soc. 14 (1982), 514-532. 5

[No] M.V. Nori: On subgroups of $G L_{n}\left(\mathbf{F}_{p}\right)$, Invent. math. 88 (1987), 257-275. 5

[PR] V. Platonov and A. Rapinchuk: Algebraic groups and number theory, Academic Press 1994. 1, 5, 5, 5, 6

[PrR] G. Prasad and A. Rapinchuk: Weakly commensurable arithmetic groups and isospectral locally symmetric spaces, Publ. Math. Inst. Hautes Études Sci. 109 (2009), 113-184. 1.3

[PS] L. Pyber and E. Szabó: Growth in finite simple groups of Lie type of bounded rank, preprint (2010), arXiv:1005.1858v1 5.10

$[\mathrm{SaV}] \quad$ A. Salehi Golsefidy and P. Varjú: Expansion in perfect groups, preprint (2010). 5.10

[Sa] L. Saloff-Coste: Random walks on finite groups, in "Probability on discrete structures", 263-346, Encyclopaedia Math. Sci., 110, Springer 2004. 5

[SGA3] Schémas en groupes. III: Structure des schémas en groupes réductifs, Séminaire de Géométrie Algébrique du Bois Marie 1962/64 (SGA 3). Dirigé par M. Demazure et A. Grothendieck, Lecture Notes in Mathematics, Vol. 153, Springer-Verlag, 1962/1964. 3, 3

[Sp] T.A. Springer: Linear algebraic groups, 2nd edition, Progr. Math. 9, Birkhaüser 1998. 1

[St1] R. Steinberg: Regular elements of semisimple algebraic groups, Publ. Math. I.H.É.S, 25 (1965), 49-80. 2.3, 4.1

[W] B. Weisfeiler: Strong approximation for Zariski-dense subgroups of semi-simple algebraic groups, Annals of Math. 120 (1984), 271-315. 5

Département de Mathématiques, Bâtiment 425, Faculté des Sciences d'Orsay, Université Paris-Sud 11, F-91405 Orsay Cedex, France

E-mail address: florent.jouve@math.u-psud.fr

ETH ZÜRICH - DMATH, RÄMISTRAsse 101, 8092 ZürICH, Switzerland

E-mail address: kowalski@math.ethz.ch

Department of Mathematics and Statistics, Queen's University, Kingston, On K7L 3N6, Canada

E-mail address: zywina@mast.queensu.ca

$U R L:$ http://www.mast.queensu.ca/ zywina 